\title{
Beta Kumaraswamy Burr Type X Distribution and Its Properties.
}

\author{
Umar Yusuf Madaki ${ }^{\mathrm{a}, \mathrm{b}, *}$, Mohd Rizam Abu Bakar ${ }^{\mathrm{a}, \mathrm{c}}$, Laba Handique ${ }^{\mathrm{d}}$ \\ ${ }^{a}$ Department of Mathematics, Faculty of Science, Universiti Putra Malaysia, Malaysia. \\ ${ }^{b}$ Department of Mathematics and Statistics, Faculty of Science, Yobe State \\ University-Nigeria. \\ ${ }^{c}$ Laboratory of Computational Statistics and Operations Research, Institute for \\ Mathematical Research, Universiti Putra Malaysia. \\ ${ }^{d}$ Department of Statistics, Faculty of Science, Dibrugarh University, India.
}

\begin{abstract}
We proposed a so-called Beta Kumaraswamy Burr Type X distribution which gives the extension of the Kumaraswamy-G class of family distribution. Some properties of this proposed model were provided, like: the expansion of densities and quantile function. We considered the Bayes and maximum likelihood methods to estimate the parameters and also simulate the model parameters to validate the methods based on different set of true values. Some real data sets were employed to show the usefulness and flexibility of the model which serves as generalization to many sub-models in the fields of engineering, medical, survival and reliability analysis.
\end{abstract}

Keywords: Bayesian, Beta Kumaraswamy-G, Burr Type X,

Kumaraswamy-G, quantile function, maximum likelihood estimation, inverse CDF method of simulation.

\section{Introduction}

Here, we provide a brief explanation on the Beta distribution which has been receiving more attention in the area of statistical modeling over a century [1]. The beta distribution has PDF and CDF defined as:

$$
\begin{aligned}
f^{B e t a}(t ; \nu, \kappa) & =\frac{1}{B(\nu, \kappa)} t^{(\nu-1)}(1-t)^{(\kappa-1)}, t \epsilon(0,1) \\
F^{B e t a}(t ; \nu, \kappa) & =I_{t}(\nu, \kappa) \quad t \epsilon(0,1) .
\end{aligned}
$$

\footnotetext{
Madaki et. al 2018

* Umar Yusuf Madaki

Email addresses: bomayu84@gmail.com ( Umar Yusuf Madaki), mrizam@upm.edu.my (Mohd Rizam Abu Bakar), handiquelaba@gmail.com ( Laba Handique)
} 
where the shape parameters, $\nu$ and $\kappa$, are positive. Its functions are unimodal, uni-anti modal, bathtub or constant depending on the parameter values. Beta models are adaptable in-terms of versatility and this flexibility motivates its usage in many applications. On the other side, we have Kumaraswamy (Kum) distribution [2], which fails to accept the fact that Beta model does not fit hydrological data. The PDF and $\operatorname{CDF}$ of the $\operatorname{Kum} \operatorname{Model}, \operatorname{Kum}(\varphi, \psi)$, is given as:

$$
\begin{aligned}
& f^{K u m}(t ; \varphi, \psi)=\varphi, \psi t^{(\varphi-1)}\left(1-t^{\varphi}\right)^{(\psi-1)} t \epsilon(0,1) \\
& F^{K u m}(t ; \varphi, \psi)=1-\left(1-t^{\varphi}\right)^{(\psi)} t \epsilon(0,1)
\end{aligned}
$$

\subsection{Beta-Generated (Beta-G) Family of Distribution}

Beta-G family was proposed by [3] and [31] is a very versatile and rich class of generalized distributions. It recieves a quite well response over the recent years. It has a PDF and CDF as follows:

$$
\begin{aligned}
f^{B G}(t ; \nu, \kappa) & =\frac{1}{B(\nu, \kappa)} f(t) F(t)^{(\nu-1)} S(t)^{(\kappa-1)}, \\
F^{B G}(t ; \nu, \kappa) & =\frac{1}{B(\nu, \kappa)} \int_{0}^{F(t)} W^{(\nu-1)}(1-W)^{(\kappa-1)} d W=I_{F(t)}(\nu, \kappa) .
\end{aligned}
$$

where $S(t)$ is the survival function of Beta-G distribution. Some of the well known works related to Beta-G family of distributions received an impact for a given distribution with $\mathrm{PDF}$ and $\mathrm{CDF}$ of Beta-G proposed by [3], [4] and [5] family of distribution.

\subsection{Kumaraswamy-G (Kum-G) Family of Distribution:}

Kumaraswamy-G family received a quite well attention in the recent years in the area of applied statistics proposed by [6], this family called Kum-G family motivate us in which we applied the method which proposed by [7] in developing our model having PDF and CDF define respectively as:

$$
\begin{aligned}
f^{K u m-G}(t ; \varphi, \psi) & =\varphi \psi g(t) G(t)^{(\varphi-1)}\left[1-G(t)^{\varphi}\right]^{(\psi-1)}, \\
F^{K u m-G}(t ; \varphi, \psi) & =1-\left[1-G(t)^{\varphi}\right]^{\psi}
\end{aligned}
$$

where, $t>0, g(t)=\frac{d}{d t} G(t)$ and $\varphi, \psi>0$, denotes the shape parameters on the notion for other parameters in the baseline distribution. Kumaraswamy-G class by [6], received a quite well attention to researchers in the last few decades in the literature.

\subsection{Beta Generated Kumaraswamy-G (Beta Kum-G) Family of Distribution:}

Recently [8]-[32], proposed this new family of distribution called Beta Generated Kumaraswamy-G (Beta Kum-G) distribution having $\theta=p, q \varphi, \psi, \vartheta$ and $\tau$ where they adopt the method of Beta-Generator proposed by [3] and reviewed by [4]-[5]. This new family called beta Kum-G family motivate us in which we 
applied the method by [7], in developing our model. It has a PDF and CDF given as:

$$
\begin{aligned}
f^{B K u m-G}(t ; \nu, \kappa, \varphi, \psi)= & \frac{1}{B(\nu, \kappa)} \varphi \psi g(t) G(t)^{(\varphi-1)} \\
& \times\left[1-G(t)^{\varphi}\right]^{(\psi \kappa-1)}\left[1-\left\{1-G(t)^{\varphi}\right\}^{\psi}\right]^{(\nu-1)}, \\
F^{B K u m-G}(t ; \nu, \kappa, \varphi, \psi)= & I_{1-\left[1-G(t)^{\varphi}\right]^{\psi}}(\nu, \kappa) .
\end{aligned}
$$

This new family can also be referred to as the new generalized Kumaraswamy-G (GKum-G) family of distribution.

\subsection{Burr Type $X(B X)$}

This model was proposed by [9] and revisited by [10]-[11], whom contribute a lot to family of continuous distributions and played a vital role in medical, survival and reliability analysis. Our first motivation arises to the failure or hazard rate function having some important features including the Burr-Type $\mathrm{X}(\mathrm{BX})$ property which exhibits the fitting of engineering and medical data sets, more especially big data, as it was recently observed by [12], that BX model is very effective and versatile in modeling reliability strength and lifetime data sets. They also thought BX model can be exponentiated Rayleigh (ER) or generalized Rayleigh (GR), but they prefer calling it BX model due to its suitability to Burr family of distribution and properties. They observed that the GR or BX model with two-parameter also has a quite common properties with gamma, generalized exponential and Wei-bull distributions respectively. Although, the BX model density and cumulative distribution functions have a simple close form and its also has a convenient and flexible feature in modeling censored (incomplete) data, unlike gamma, GE and Weibull distributions. The twoparameter BX has a monotonically increasing and decreasing hazard function features, which can be used for practical aspects in statistical distribution and modeling of applications. Recently, authors have been studying BX model due to its ability and flexibility in modeling reliability data sets such as, [13] whom proposed the two parameter Burr type X distribution which authors like; [14] and [15] made some extension. The Burr type $\mathrm{X}$ distribution can be used in modeling general lifetime data. Based on this model distribution in [10], whose PDF and CDF are given by:

$$
\begin{aligned}
& f_{B X}(t ; \vartheta, \tau)=2 \vartheta \tau^{2} t e^{-(\tau t)^{2}}\left\{1-e^{-(\tau t)^{2}}\right\}^{(\vartheta-1)}, \\
& F_{B X}(t ; \vartheta, \tau)=\left\{1-e^{-(\tau t)^{2}}\right\}^{\vartheta} .
\end{aligned}
$$

These two Figure (1) and Figure (2), were originally plotted by [11], showing the flexibility (left-skewed, right-skewed and symmetry), shapes (bathtub and increasing) respectively. The two parameters BX or GR model in equation (11) above, implies $B X(\vartheta, \tau)$, if $\vartheta=1$, BX distribution reduces to a well-known one parameter Rayleigh distribution. Where $\vartheta$ and $\tau$ are the shape and scale parameters respectively. The two parameters BX or GR model in equation 11 


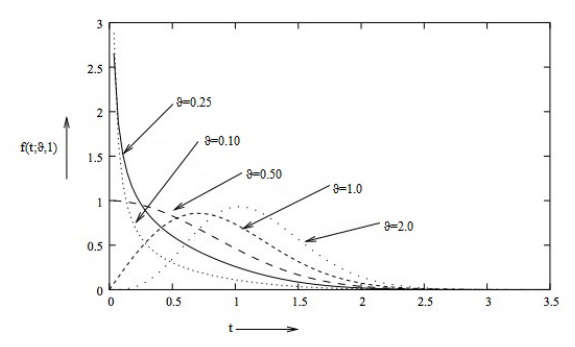

Figure 1: Plot of the Burr Type X probability density function for the shape parameter $\vartheta$.

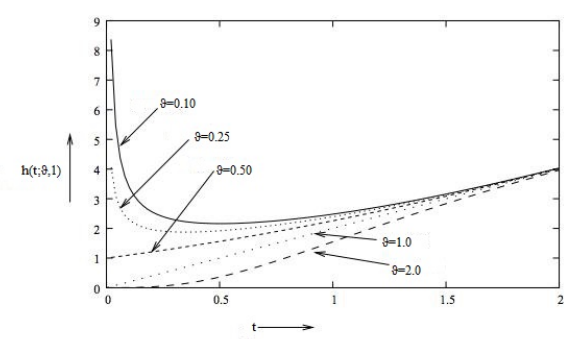

Figure 2: Plot of the Burr Type X hazard function for the shape parameter $\vartheta$.

above, implies $B X(\vartheta, \tau)$, if $\vartheta=1$, BX distribution reduces to a well-known one parameter Rayleigh distribution. If $\vartheta \neq 1$, this plays the role of $\tau$, which is the scale parameter. On the other hand, if $\vartheta \leq \frac{1}{2}$, therefore the PDF of BX model will be decreasing function of the model while for $\vartheta>\frac{1}{2}$, it proves that it is a unimodal right skewed function. If it has a mode written as $\frac{t_{o}}{\tau}$, where $t_{o}$ is called the non-linear systems of equation given as:

$$
1-2 t^{2}-e^{-t^{2}}\left(1-2 \vartheta t^{2}\right)=0 .
$$

The above mode of BX model shows clearly that it is decreasing function of the scale parameter $\tau$ and also the increasing function of $\vartheta$ respectively. The PDF figures at different forms resemble that of Weibull and gamma functions. Moreover, the median of the BX model occurs when the given quantile at:

$$
\left[-\frac{1}{\tau} \ln \left(1-\frac{1}{2}\right)^{\frac{1}{\vartheta}}\right]^{\frac{1}{2}}
$$

This also shows that the non-increasing form of $\tau$, and the non-decreasing form of $\vartheta$. The remaining part of the section were organized as follows. In section 2 , we provide the distributional properties, the expansions for the PDF and CDF. In section 3, the expansion of densities, sub-models. In Section 4, we obtain the moments, moment generating function and the order statistics, Re' nyi entropy, the quantile function, Skewness and Kurtosis and maximum likelihood 
estimation. In section 5, application of real data and comparison of the new model with some sub-models was done and follow by the conclusion in section ?? respectively.

\section{Distributional Properties}

\subsection{Beta Kumaraswamy Burr Type X (Beta Kum-BX) Distribution:}

In this section, we present our new model Beta Kum-BX with six parameters as the properties. Our second motivation, lies within the wide usage of Kum-G family define in equation (7) and the baseline distribution known as the BX define in equation (11) together with the property of beta-G family define in equation (5) and (6) above due the relative flexibility and capability in modeling agriculture, engineering and medical datasets in respect to the model suitability at different tractable or complex situations. However, we were motivated to introduced this new model called Beta Kumaraswamy Burr Type X (Beta Kum$\mathrm{BX}$ ) with six parameters $\theta=\nu, \kappa, \varphi, \psi, \vartheta$ and $\tau$ by confounding equation (9) and (11) and also equation (10) and (12) by the methods of beta- $G$ generator proposed by [8] and thereby Burr Type $\mathrm{X}$ as the baseline distribution which we obtain the probability density function PDF given as:

$$
\begin{aligned}
f^{B K B X}(t ; \theta)= & \frac{2}{B(\nu, \kappa)} \varphi \psi \vartheta \tau^{2} t e^{-(\tau t)^{2}}\left\{1-e^{-(\tau t)^{2}}\right\}^{(\vartheta \varphi-1)} \\
& \times\left[1-\left\{1-e^{-(\tau t)^{2}}\right\}^{\vartheta \varphi}\right]^{\psi \kappa-1} \\
& \times\left[1-\left[1-\left\{1-e^{-(\tau t)^{2}}\right\}^{\vartheta \varphi}\right]^{\psi}\right]^{\nu-1} .
\end{aligned}
$$

We now show that the $\int_{0}^{t} f(t) d t=1$. Therefore from equation (13), above we simplify the model and proof the theorem.

$$
\begin{gathered}
\text { Hence, let } M=\left[1-\left\{1-e^{-(\tau t)^{2}}\right\} \vartheta \varphi \psi\right] \text { and } \\
d_{M}=2 \varphi \psi \vartheta \tau^{2} t e^{-(\tau t)^{2}}\left\{1-e^{-(\tau t)^{2}}\right\}^{(\vartheta \varphi-1)}
\end{gathered}
$$

Therefore, equation (13), becomes more like a complete beta function given as;

$$
\begin{gathered}
\int_{0}^{t} f(t) d t=\frac{1}{B(\nu, \kappa)} \int_{0}^{t} M^{(\kappa-1)}(1-M)^{(\nu-1)} d_{M}, \\
\text { where, } \int_{0}^{t} M^{(\kappa-1)}(1-M)^{(\nu-1)} d_{M}=B(\nu, \kappa) \\
\text { Hence, } \frac{1}{B(\nu, \kappa)} \times B(\nu, \kappa)=1 .
\end{gathered}
$$

The corresponding cumulative distribution function, survival function $S(t)$, hazard or failure rate function $h(t)$ and cumulative hazard function $H(t)$ respec- 
tively.

$$
\begin{aligned}
& F^{B K B X}(t ; \theta)=I_{\left.\left[1-\left(1-e^{-(\tau t)^{2}}\right)^{\vartheta} \varphi\right]^{\psi}\right]}(\nu, \kappa), \\
& S^{B K B X}(t ; \theta)=1-F^{B K B X}(t), \\
& =1-I_{\left.1-\left\{\left(1-e^{-(\tau t)^{2}}\right)^{\vartheta \varphi}\right\}^{\psi}\right]}(\nu, \kappa), \\
& h^{B K B X}(t ; \theta)=\frac{2}{\left\{\left(1-e^{-(\tau t)^{2}}\right)^{\vartheta \varphi}\right\}^{\psi} B(\nu, \kappa)} \text {, } \\
& \times \varphi \psi \vartheta \tau^{2} t e^{-(\tau t)^{2}}\left\{1-e^{-(\tau t)^{2}}\right\}^{(\varphi-1)} \\
& \times\left[1-\left\{1-e^{\left.-(\tau t)^{2}\right)^{\vartheta \varphi}}\right\}\right]^{\psi \kappa-1}, \\
& \times\left[1-\left[1-\left\{1-e^{-(\tau t)^{2}}\right\}^{\vartheta \varphi}\right]^{\psi}\right]^{\kappa-1} \\
& H^{B K B X}(t ; \theta)=-\ln \left[\left\{\left(1-e^{-(\tau t)^{2}}\right)^{\vartheta \varphi}\right\}^{\psi}\right] .
\end{aligned}
$$

Based on the relative usage of Kumaraswamy and Burr Type X baseline distributions we were motivated to propose this six parameters Beta Kum-BX due to the wideness of the BX with two parameters which generalizes (Rayleigh) and it provides a continuous function among the twelve Burr family of distribution which makes it suitable in modeling several complex situations. Also, the model transformation in equation (14) are not submissive meaning not mostly tractable just like the Weibull distribution where the skewness and kurtosis at different shapes were flexible with monotonically non-decreasing and non-increasing hazard functions.

Our last motivation was noted from the above CDF of the new model equation (14), $F_{\text {BetaKum-BX }}(t, \theta) m_{1} Y$ as $t \rightarrow 0$ also $1-F_{\text {BetaKum-BX }}(t, \theta) \sim$ $m_{2} \exp -(\tau t)^{2}$ as $t \rightarrow \infty$ where $m_{1}$ and $m_{2}$ denotes the model constraints. This new model BKBX encloses a lower tail performance for the exponentiated Rayleigh model and the upper tail acts like the Burr-Type X model. Kumaraswamy Burr-Type X which are the (Kumaraswamy and Burr-Type X) motivates us with its great varieties of fitting failure, engineering (reliability), medicine and agriculture etc. This six parameter model encompasses many sub-models with great flexibility which in their bathtub shapes, monotonically non-increasing and decreasing failure function and also has some common properties with Kumaraswamy-Weibull by [18] which fit more perfectly well than the reduce modified Kumaraswamy Burr type X model relating to flexibility and suitability towards multivariate and censored data respectively.

Figure (3) and Figure (4), describes the shapes of the probability density function and cumulative distribution function functions for the given parameter values. These function represent different kind of forms depending on choosing values of BKBX model parameters. We noticed that the additional shape parameters allows for high level of flexibility.

\subsection{Shapes of the BKBX Distribution}

On the other hand, Figure (5) and Figure (6) below, shows the failure rate or hazard function of BKBX model increasing and decreasing or bathtub shapes. 


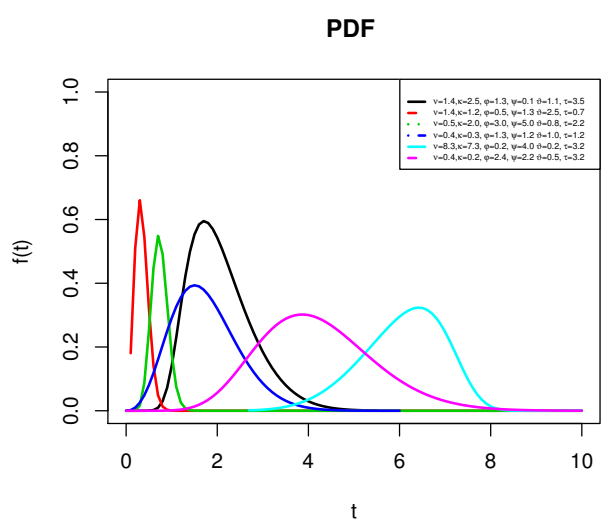

Figure 3: Plot of the BKBX probability density function (PDF) for different parameter values of $\nu, \kappa, \varphi, \psi, \vartheta$ and $\tau$.

This new model with six parameters is more flexible than Kumaraswamy Burr Type X and Burr Type X distribution with four and two parameters due to flexibility of Kumaraswamy distribution with two parameters, that leads more smooth and vital. This new model will be useful in modeling and analyzing real life, censored and uncensored data in medical, engineering, pure science and agricultural areas. 


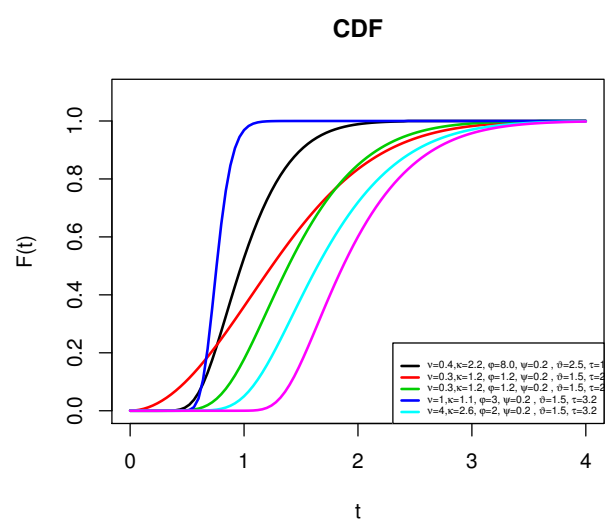

Figure 4: Plot of the BKBX cumulative distribution function (CDF) for different parameter values of $\nu, \kappa, \varphi, \psi, \vartheta$ and $\tau$.

\section{Expansion of PDF and CDF}

We provide expansion of the PDF of BKBX distribution in terms of infinite weighted sum. We obtain some structural properties based on the pdf expansions such as moment, moment generating function, mean division and others. The expansion is being done by using the equation of the PDF from equation (13). According to [6], if $|W|<1$ and $\omega>0$ is a real non-integer, we have the series representation :

$$
(1-W)^{\omega-1}=\sum_{j=0}^{\infty}(-1)^{j}\left(\begin{array}{c}
\omega-1 \\
j
\end{array}\right) W^{j}
$$

If $\omega$ is an integer number, the series representation for finding CDF expansion is:

$$
(1-W)^{\omega-1}=\sum_{j=0}^{\omega-1}(-1)^{j}\left(\begin{array}{c}
\omega-1 \\
j
\end{array}\right) W^{j}
$$

By using the expansion of equation (18) and $\omega>0$ real non-integer, By using the binomial expansion in equation (13), above which is the density function of BKBX distribution with six parameters, $\theta=(\nu, \kappa, \varphi, \psi, \vartheta, \tau)$ and $\theta_{2}=$ 


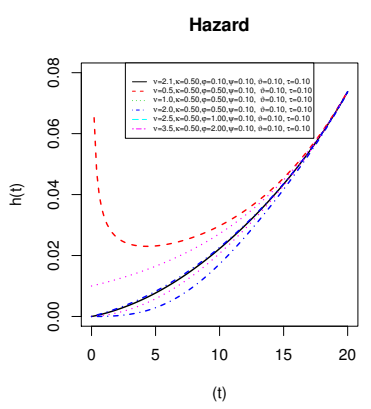

(a) Hazard Function 1.

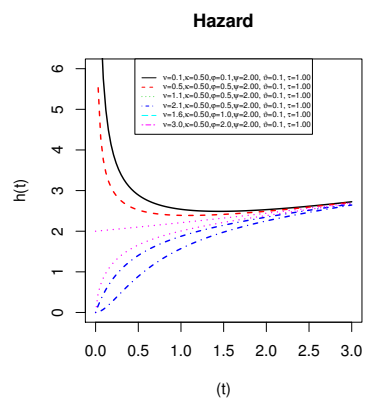

(b) Hazard Function 2.

Figure 5: Plot of the BKBX Hazard function for different parameter values of $\nu, \kappa, \varphi, \psi, \vartheta$ and $\tau$.

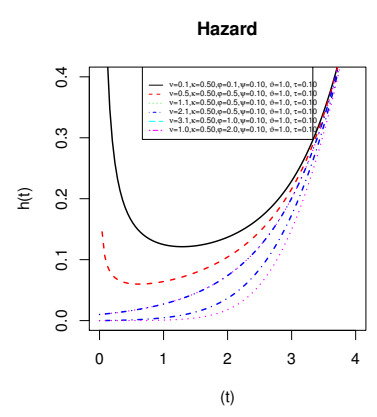

(a) Hazard Function 3.

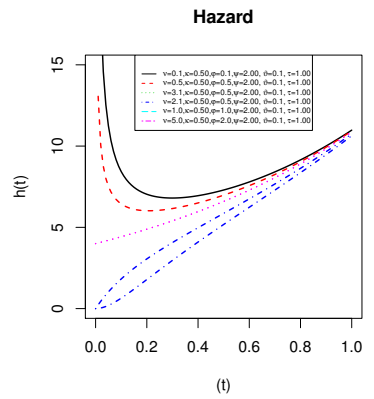

(b) Hazard Function 4 .

Figure 6: Plot of the BKBX Hazard function for different parameter values of $\nu, \kappa, \varphi, \psi, \vartheta$ and $\tau$. 
$(\varphi, \psi, \vartheta, \tau)$. We denote the following:

$$
\begin{aligned}
M_{i}= & 1-e^{-(\tau t)^{2}}, \\
R_{i}= & {\left[1-(M)^{\vartheta \varphi}\right] . } \\
f^{B K B X}(t ; \theta)= & \frac{2}{B(\nu, \kappa)} \varphi \psi \vartheta \tau^{2} t e^{-(\tau t)^{2}}\left[M_{i}\right]^{(\vartheta \varphi-1)}\left[R_{i}\right]^{\psi \kappa-1}, \\
& \times \sum_{j=0}^{\nu-1}(-1)^{j}\left(\begin{array}{c}
\nu-1 \\
j
\end{array}\right)\left[1-\left(1-e^{-(\tau t)^{2}}\right)^{\varphi}\right]^{\psi_{j}}, \\
= & \sum_{j=0}^{\nu-1}(-1)^{j}\left(\begin{array}{c}
\nu-1 \\
j
\end{array}\right) \dot{\psi}_{j} f^{K B X}\left(t ; \theta_{2}(j+n)\right), \\
\text { Where, } \hat{\psi}_{j}= & \frac{(-1)^{j+1}}{B(\nu, \kappa)(j+n)}\left(\begin{array}{c}
\nu-1 \\
j
\end{array}\right) .
\end{aligned}
$$

Adjusting further we obtained from Equation (19).

$$
\begin{aligned}
& \left.=\sum_{j=0}^{\nu-1} \dot{\psi}_{j} \frac{d}{d t} F^{K B X}\left(t ; \theta_{2}(j+n)\right)\right] \\
& \left.=\sum_{j=0}^{\nu-1} \dot{\psi}_{j} \frac{d}{d t} F^{K B X}\left(t ; \theta_{2}\right)\right]^{(j+n)} \\
& \left.=f^{K B X}\left(t ; \theta_{2}\right) \sum_{j=0}^{\nu-1} \dot{\psi}_{j} F^{K B X}\left(t ; \theta_{2}\right)\right]^{(j+n-1)}
\end{aligned}
$$

Where, $\dot{\psi}_{j}=\psi_{j}(j+n)$ Alternatively, we can expand the PDF also used by [6]:

$$
\begin{aligned}
& f^{B K B X}(t ; \theta)=f^{K B X}\left(t ; \theta_{2}\right) \sum_{j=0}^{\nu-1} \dot{\psi}_{j}\left[F^{K B X}\left(t ; \theta_{2}\right)\right]^{(j+n-1)}, \\
& =f^{K B X}\left(t ; \theta_{2}\right) \sum_{j=0}^{\nu-1} \hat{\psi}_{j}\left[1-F^{K B X}\left(t ; \theta_{2}\right)\right]^{(j+n-1)}, \\
& =f^{K B X}\left(t ; \theta_{2}\right) \sum_{j=0}^{\nu-1}\left\{\hat{\psi}_{j}(j+n)\right\}\left[F^{K B X}\left(t ; \theta_{2}\right)\right]^{(j+n-1)} \text {, } \\
& =f^{K B X}\left(t ; \theta_{2}\right) \sum_{j=0}^{\nu-1} \dot{\psi}_{j} \sum_{l=0}^{j+n-1}\left(\begin{array}{c}
j+n-1 \\
l
\end{array}\right)(-1)^{l}\left[F^{K B X}\left(t ; \theta_{2}\right)\right]^{l} \text {, } \\
& =f^{K B X}\left(t ; \theta_{2}\right) \sum_{j=0}^{\nu-1} \eta_{l}\left[F^{K B X}\left(t ; \theta_{2}\right)\right]^{l},
\end{aligned}
$$

Where, $\eta_{l}=\psi_{j} \sum_{l=0}^{j+n-1} \cdot(-1)^{l}\left(\begin{array}{c}j+n-1 \\ l\end{array}\right)$. 
Also we can expand the CDF with the following result using an incomplete beta function also provided by [5].

$$
I_{y}(\varphi, \psi, \vartheta, \tau)=\frac{2 y^{\vartheta \varphi}}{B(\nu, \kappa)} \sum_{\kappa=0}^{\infty}\left(\begin{array}{c}
\psi-1 \\
\kappa
\end{array}\right) \frac{(-1)^{\kappa} y^{\kappa}}{\tau(\varphi+\kappa)} .
$$

Also, on the other hand, we have the CDF of BKBX as:

$$
\left.F^{B K B X}(t ; \theta)=1-\left[1-\left(1-e^{-(\tau t)^{2}}\right)^{\vartheta \varphi}\right]^{\psi}\right]^{(\nu, \kappa)} .
$$

Using Equation (24), above we obtained:

$$
\begin{aligned}
& =\frac{2}{B(\nu, \kappa)}\left[1-\left[1-\left(1-e^{-(\tau t)^{2}}\right)^{\vartheta \varphi}\right]^{\psi}\right]^{\nu} \sum_{i=0}^{\infty}\left(\begin{array}{c}
\kappa-1 \\
i
\end{array}\right) \frac{(-1)^{i}}{\nu+i}\left[1-\left[1-\left(1-e^{-(\tau t)^{2}}\right)^{\vartheta \varphi}\right]^{\psi}\right]^{i}, \\
& =\sum_{i=0}^{\infty} \frac{(-2)^{i}}{B(\nu, \kappa)(\nu+i)}\left(\begin{array}{c}
\kappa-1 \\
i
\end{array}\right)\left[F^{K B X}\left(t ; \theta_{2}\right)^{\nu+i}\right] \\
& \left.=\sum_{i=0}^{\infty} \frac{(-2)^{i}}{B(\nu, \kappa)(\nu+i)}\left(\begin{array}{c}
\kappa-1 \\
i
\end{array}\right) \sum_{j=0}^{\infty}\left(\begin{array}{c}
\nu+i \\
j
\end{array}\right)(-1)^{j} F^{K B X}\left(t ; \theta_{2}\right)\right]^{j} \\
& =\sum_{i, j=0}^{\infty} \frac{(-2)^{i+j}}{B(\nu, \kappa)(\nu+i)}\left(\begin{array}{c}
\kappa-1 \\
i
\end{array}\right)\left(\begin{array}{c}
\nu+i \\
j
\end{array}\right) \sum_{k=0}^{j}\left(\begin{array}{l}
j \\
k
\end{array}\right)(-1)^{k}\left[F^{K B X}\left(t ; \theta_{2}\right)\right]^{k} \\
& =\sum_{i, j=0}^{\infty} \sum_{k=0}^{j} \frac{(-2)^{i+j+k}}{B(\nu, \kappa)(\nu+i)}\left(\begin{array}{c}
\kappa-1 \\
i
\end{array}\right)\left(\begin{array}{c}
\nu+i \\
j
\end{array}\right)\left(\begin{array}{l}
j \\
k
\end{array}\right)\left[F^{K B X}\left(t ; \theta_{2}\right)\right]^{k}
\end{aligned}
$$

By exchanging the indices $\mathrm{j}$ and $\mathrm{k}$ in the symbol, we get:

$$
\begin{aligned}
& =\sum_{i, k=0}^{\infty} \sum_{j=k}^{\infty} \frac{(-2)^{i+j+k}}{B(\nu, \kappa)(\nu+i)}\left(\begin{array}{c}
\kappa-1 \\
i
\end{array}\right)\left(\begin{array}{c}
\nu+i \\
j
\end{array}\right)\left(\begin{array}{l}
j \\
k
\end{array}\right)\left[F^{K B X}\left(t ; \theta_{2}\right)\right]^{k}, \\
& =\sum_{k=0}^{\infty} \mu_{k}\left[F^{K B X}\left(t ; \theta_{2}\right)\right]^{k}, \\
\text { Where, } \mu_{k} & =\sum_{i=0}^{\infty} \sum_{j=k}^{\infty} \frac{(-2)^{i+j+k}}{B(\nu, \kappa)(\nu+i)}\left(\begin{array}{c}
\kappa-1 \\
i
\end{array}\right)\left(\begin{array}{c}
\nu+i \\
j
\end{array}\right)\left(\begin{array}{l}
j \\
k
\end{array}\right) .
\end{aligned}
$$

On the other hand, the expansion of the CDF of BKBX can be derived similarly as provided by [33], 


$$
\begin{aligned}
= & {\left.\left[1-\left(1-\left(1-e^{-(\tau t)^{2}}\right)^{\vartheta \varphi}\right]^{\psi}\right)(\nu, \kappa)\right]=\left[F^{B K B X}(t ; \theta)\right] } \\
= & \sum_{m=\nu}^{\nu+\kappa-1}\left(\begin{array}{c}
\nu+\kappa-1 \\
m
\end{array}\right)\left[F^{K B X}\left(t ; \theta_{2}\right)\right]^{m}\left[F^{K B X}\left(t ; \theta_{2}\right)\right]^{(\nu+\kappa-1-m)}, \\
= & \sum_{m=\nu}^{\nu+\kappa-1}\left(\begin{array}{c}
\nu+\kappa-1 \\
m
\end{array}\right)\left[F^{K B X}\left(t ; \theta_{2}\right)\right]^{m} \\
& \times \sum_{n=0}^{\nu+\kappa-1-m}\left(\begin{array}{c}
\nu+\kappa-1-m \\
n
\end{array}\right)\left[F^{K B X}\left(t ; \theta_{2}\right)\right]^{n} \cdot \\
= & \sum_{m=\nu}^{\nu+\kappa-1} \sum_{n=0}^{\nu+\kappa-1-m}\left(\begin{array}{c}
\nu+\kappa-1 \\
m
\end{array}\right)(\nu+\kappa-1-m \\
= & \left.\sum_{m=\nu}^{\nu+\kappa-1} \sum_{n=0}^{\nu+\kappa-1-m} \eta_{l}\left[F^{K B X}\left(t ; \theta_{2}\right)\right]^{m+n} \cdot F^{K B X}\left(t ; \theta_{2}\right)\right]^{(m+n)} \\
& (27)
\end{aligned}
$$

Where, $\eta_{l}=\left(\begin{array}{c}\nu+\kappa-1 \\ m\end{array}\right)\left(\begin{array}{c}\nu+\kappa-1-m \\ n\end{array}\right)(-1)^{n}$.

\subsection{Some Special Sub-models}

The BKBX with $\theta=(\nu, \kappa, \varphi, \psi, \vartheta, \tau)$ has several special cases or sub models but among are few stated below:

1. When $\varphi=\psi=1$, the BKBX in Equation (13) above reduces to Beta Burr Type $\mathrm{X}$ with four parameters by [14].

\section{Show that equation (13) reduces to Beta Burr Type X with} four parameters

Let $\varphi=\psi=1$ in equation (13), given as;

$$
\begin{aligned}
f^{B K B X}(t ; \theta)= & \frac{2}{B(\nu, \kappa)}(1)(1) \vartheta \tau^{2} t e^{-(\tau t)^{2}}\left\{1-e^{-(\tau t)^{2}}\right\}^{(\vartheta(1)-1)} \\
& \times\left[1-\left\{1-e^{-(\tau t)^{2}}\right\}^{\vartheta(1)}\right]^{(1) \kappa-1} \\
& \times\left[1-\left[1-\left\{1-e^{-(\tau t)^{2}}\right\}^{\vartheta(1)}\right]^{(1)}\right]^{\nu-1}
\end{aligned}
$$

We can as well reduce to BBX model as follows:

$$
\begin{aligned}
f^{B B X}(t ; \nu, \kappa, \vartheta, \tau)= & \frac{2}{B(\nu, \kappa)} \vartheta \tau^{2} t e^{-(\tau t)^{2}}\left\{1-e^{-(\tau t)^{2}}\right\}^{(\vartheta-1)} \\
& \times\left[1-\left\{1-e^{-(\tau t)^{2}}\right\}^{\vartheta}\right]^{\kappa-1} \\
& \times\left[1-\left(e^{-(\tau t)^{2}}\right)^{\vartheta}\right]^{\nu-1}
\end{aligned}
$$


Hence,

$$
\begin{aligned}
f^{B B X}(t ; \nu, \kappa, \vartheta, \tau)= & \frac{2 \vartheta \tau^{2} t e^{-(\tau t)^{2}}}{B(\nu, \kappa)}\left[1-\left(e^{-(\tau t)^{2}}\right)\right]^{(\vartheta \nu-1)} \\
& \times\left[1-\left\{1-e^{-(\tau t)^{2}}\right\}^{\vartheta}\right]^{\kappa-1}
\end{aligned}
$$

2. When $\nu=\kappa=1$, the BKBX in Equation (13) above reduces to $\mathrm{Ku}-$ maraswamy Burr Type X (KBX) with four parameters from the family of Kumaraswamy-G proposed by [6] also a special case to KumaraswamyWeibull distribution proposed by [14] respectively.

Show that equation (13) reduces to Kumaraswamy Burr Type $\mathrm{X}$ with four parameters Let $\nu=\kappa=1$ in equation (13), given as;

$$
\begin{aligned}
f^{B K B X}(t ; \theta)= & \frac{2}{B(1,1)} \varphi \psi \vartheta \tau^{2} t e^{-(\tau t)^{2}}\left\{1-e^{-(\tau t)^{2}}\right\}^{(\vartheta \varphi-1)} \\
& \times\left[1-\left\{1-e^{-(\tau t)^{2}}\right\}^{\vartheta \varphi}\right]^{(\psi(1)-1)} \\
& \times\left[1-\left[1-\left\{1-e^{-(\tau t)^{2}}\right\}^{\vartheta \varphi}\right]^{\psi}\right]^{1-1} .
\end{aligned}
$$

Hence, by law of indices let $A=\left[1-\left[1-\left\{1-e^{-(\tau t)^{2}}\right\}^{\vartheta \varphi}\right]^{\psi}\right]$ and $A^{0}=1$.

$$
\begin{aligned}
= & \frac{2}{B(1,1)} \varphi \psi \vartheta \tau^{2} t e^{-(\tau t)^{2}}\left\{1-e^{-(\tau t)^{2}}\right\}^{(\vartheta \varphi-1)} \\
& \times\left[1-\left\{1-e^{-(\tau t)^{2}}\right\}^{\vartheta \varphi}\right]^{(\psi-1)} \\
& \times\left[1-\left[1-\left\{1-e^{-(\tau t)^{2}}\right\}^{\vartheta \varphi}\right]^{\psi}\right]^{0} .
\end{aligned}
$$

We can as well reduces to KBX model as follows:

$$
\begin{aligned}
f^{K B X}(t ; \varphi, \psi, \vartheta, \tau)= & 2 \varphi \psi \vartheta \tau^{2} t e^{-(\tau t)^{2}}\left\{1-e^{-(\tau t)^{2}}\right\}^{(\vartheta \varphi-1)} \\
& \times\left\{1-\left(1-e^{-(\tau t)^{2}}\right)^{(\vartheta \varphi)}\right\}^{(\psi-1)} .
\end{aligned}
$$

3. When $\varphi=\psi=1, \nu=\kappa=1$, the BKBX in Equation (13) above reduces to Burr Type X distribution with two parameters [12]-[13].

Show that equation (13) reduces to Burr Type $\mathrm{X}$ with two parameters Let $\nu=\kappa=\varphi=\psi=1$ in Equation (13), given as;

$$
\begin{aligned}
f^{B K B X}(t ; \theta)= & \frac{2}{B(1,1)} \varphi \psi \vartheta \tau^{2} t e^{-(\tau t)^{2}}\left\{1-e^{-(\tau t)^{2}}\right\}^{(\vartheta \varphi-1)} \\
& \times\left[1-\left\{1-e^{-(\tau t)^{2}}\right\}^{\vartheta \varphi}\right]^{(\psi(1)-1)} \\
& \times\left[1-\left[1-\left\{1-e^{-(\tau t)^{2}}\right\}^{\vartheta \varphi}\right]^{\psi}\right]^{1-1} .
\end{aligned}
$$

Hence, by law of indices let $A=1-\left[1-\left\{1-e^{-(\tau t)^{2}}\right\}^{\vartheta(1)}\right]$ and $A^{0}=1$, 
likewise, $B=\left[1-\left\{1-e^{-(\tau t)^{2}}\right\}^{\vartheta(1)}\right]$ and $B^{0}=1$

$$
\begin{aligned}
= & \frac{2}{B(1,1)}(1)(1) \vartheta \tau^{2} t e^{-(\tau t)^{2}}\left\{1-e^{-(\tau t)^{2}}\right\}^{(\vartheta(1)-1)} \\
& \times\left[1-\left\{1-e^{-(\tau t)^{2}}\right\}^{\vartheta(1)}\right]^{(1-1)} \\
& \times\left[1-\left[1-\left\{1-e^{-(\tau t)^{2}}\right\}^{\vartheta(1)}\right]^{(1)}\right]^{0}
\end{aligned}
$$

We can as well reduces to BX model as follows:

$$
f^{B X}(t ; \vartheta, \tau)=2 \vartheta \tau^{2} t e^{-(\tau t)^{2}}\left\{1-e^{-(\tau t)^{2}}\right\}^{(\vartheta-1)} .
$$

4. When $\nu=\kappa=\varphi=\vartheta=\tau=1$, the BKBX in Equation (13) above reduces to Rayleigh distribution with one scale parameter by [19].

Show that equation (13) reduces to Rayleigh with with one scale parameter. Let $\nu=\kappa=\varphi=\vartheta=\tau=1$ in equation (13), given as;

$$
\begin{aligned}
= & \frac{2}{B(1,1)}(1)(1)(1) \tau^{2} t e^{-(\tau t)^{2}}\left\{1-e^{-(\tau t)^{2}}\right\}^{((1)(1)-1)} \\
& \times\left[1-\left\{1-e^{-(\tau t)^{2}}\right\}^{(1)(1)}\right]^{(1)(1)-1} \\
& \times\left[1-\left[1-\left\{1-e^{-(\tau t)^{2}}\right\}^{(1)(1)}\right]^{(1)}\right]^{(1)-1} .
\end{aligned}
$$

We can as well reduces to $\mathrm{R}$ model from BX model substituting $\vartheta=1$, by the law of indices let $C=\left\{1-e^{-(\tau t)^{2}}\right\}$ and $C^{0}=1$ :

$$
=2(1) \tau^{2} t e^{-(\tau t)^{2}}\left\{1-e^{-(\tau t)^{2}}\right\}^{(0)} .
$$

Hence,

$$
f^{R}(t ; \tau)=2 \tau^{2} t e^{-(\tau t)^{2}}
$$

Figure (7) below shows the flow chart of all the sub-models of BKBX distribution at each level, where it provides how the six parameters reduces to its last submodel with one scale parameter Rayleigh (R) by [19] and one shape parameter Burr Type X (BX1) [9] respectively. 


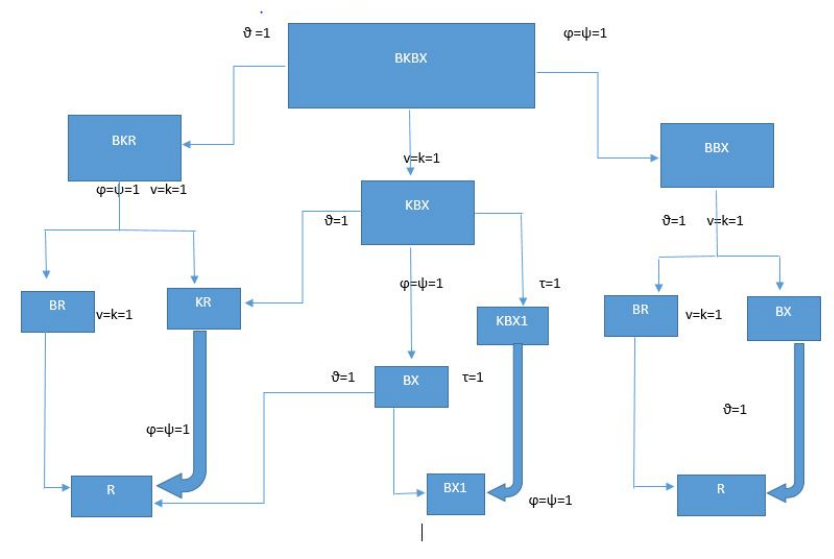

Figure 7: Sub models of BKBX Distribution

\section{Probability Weighted $r^{t h}$ Moments}

Probability weighted $r^{t h}$ moments (PWMs), It was initially introduced by [20], defined to be the expectation of some functions of a random variable $\mathrm{x}$ and y defined. The $(m, n, t))^{t h}$ PWM of $T$ is defined by:

$$
\Gamma \varphi=\int_{-\infty}^{\infty} t^{m} F(t)^{n}[1-F(t)]^{r} f(t) d t .
$$


From the above equation (23) and equation (27), the $s^{\text {th }}$ moment of $T$ can be written either as,

$$
\begin{aligned}
E\left(T^{s}\right) & =\int_{-\infty}^{\infty} t^{s} f^{K B X}\left(t ; \theta_{2}\right) d t \\
& =\sum_{j=0}^{\nu-1} \psi_{j} \int_{-\infty}^{\infty} t^{s}\left[F^{K B X}\left(t ; \theta_{2}\right)\right]^{(j+\kappa-1)} f^{K B X}\left(t ; \theta_{2}\right) d t \\
E\left(T^{s}\right) & =\sum_{j=0}^{\nu-1} \psi_{j} \Gamma_{s, 0, j+\kappa-1}, \\
\text { or, } & =\sum_{j=0}^{j+\kappa-1} \eta_{l} \int_{-\infty}^{\infty} t^{s}\left[F^{K B X}\left(t ; \theta_{2}\right)\right]^{l} f^{K B X}\left(t ; \theta_{2}\right) d t \\
E\left(T^{s}\right) & =\sum_{j=0}^{j+\kappa-1} \eta_{l} \Gamma_{s, l, 0} . \\
\text { Where, } \Gamma_{s, l, 0} & =\int_{-\infty}^{\infty} t^{s}\left[F^{K B X}\left(t ; \theta_{2}\right)\right]^{l} f^{K B X}\left(t ; \theta_{2}\right) d t \\
\eta_{l} & =\left(\begin{array}{c}
\nu+\kappa-1 \\
m
\end{array}\right)\left(\begin{array}{c}
\nu+\kappa-1-m \\
n
\end{array}\right)(-1)^{n} .
\end{aligned}
$$

is the PWM of $\operatorname{KBX} \theta_{2}=(\varphi, \psi, \vartheta, \tau)$ distribution. Therefore the moments of the BKBX $\theta=(\nu, \kappa, \varphi, \psi, \vartheta, \tau))$ can be expressed in terms of the PWMs of KBX model. The PWM method can be generally be used for estimating parameters quantiles of generalized distributions. These moments have low variance and no severe biases, also they compare favorably with estimators obtained by maximum likelihood estimator. Proceeding as the above $s^{\text {th }}$ moment of the $r^{\text {th }}$ order statistic $T_{r: q}$ in the random sample of size "n" [15].

From the order statistics in equation(31), we have,

$$
\begin{aligned}
E\left(T_{r: \kappa}^{s}\right) & =\sum_{z=0}^{k+l} \xi_{y} \Gamma_{s, 0, z} . \\
\text { Where, } \dot{\xi}_{y} & =\sum_{j=0}^{k+\kappa-1} \sum_{k=0}^{\infty} \frac{(-1)^{y+1} \gamma_{j, k}}{y+1}\left(\begin{array}{c}
k+j \\
y
\end{array}\right) . \\
\xi_{y} & =\dot{\xi}_{y}(y+1) . \\
\text { Where, } \dot{\psi}_{j} & =\frac{(-1)^{j+1}}{B(\nu, \kappa)(j+n)}\left(\begin{array}{c}
\nu-1 \\
j
\end{array}\right) . \\
\text { Where, } \eta_{l} & =\left(\begin{array}{c}
\nu+\kappa-1 \\
m
\end{array}\right)\left(\begin{array}{c}
\nu+\kappa-1-m \\
n
\end{array}\right)(-1)^{n} .
\end{aligned}
$$


These follows an incomplete gamma function provided by [14].

$$
\begin{aligned}
\Gamma \varphi & =\int_{0}^{\infty} e^{-y} y^{\varphi-1} d y \\
E\left(X^{r}\right) & =\frac{\varphi \psi \vartheta \Gamma(r / 2+1)}{B(\nu, \kappa) \tau^{r}} C_{1} \frac{1}{(k+1)^{\frac{r}{2}+1}} . \\
\text { Where, } C_{1} & =\sum_{i=0}^{\nu-1} \sum_{j=0}^{m(i+n)-j \vartheta \varphi(j+1)} \sum_{k=0}^{m}(-1)^{i+j+k}\left(\begin{array}{c}
\nu-1 \\
i
\end{array}\right)\left(\begin{array}{c}
m(i+n)-j \\
j
\end{array}\right) .
\end{aligned}
$$

\subsection{Moment Generating Function (MGF)}

The MGF of BKBX distribution can be obtained and expressed in form of exponential Kum-G family of distribution from the results we obtained in the moments above using Equation 20 above,

$$
\begin{aligned}
& M_{T}(s)=E\left[e^{s} t\right]=\int_{-\infty}^{\infty} e^{s} t f(t ; \theta) d t \\
& =\sum_{j=0}^{\nu-1} \psi_{j} \int_{-\infty}^{\infty} e^{s} t \frac{d}{d t}\left[F^{K B X}\left(t ; \theta_{2}\right)\right]^{(j+n)} d t \\
& =\sum_{j=0}^{\nu-1} \psi_{j} M_{X}(s), \\
& \text { Where, } M_{X}(s) . \\
& =\int_{-\infty}^{\infty} e^{s} t \frac{d}{d t}\left[F^{K B X}\left(t ; \theta_{2}\right)\right]^{(j+n)} d t
\end{aligned}
$$

Where $M_{X}(s)$ is the MGF of a BKBX distribution.

\subsection{Order Statistics}

These two notable statisticians [10] and [11], proposed the procedure of deriving the order statistics of Burr Type $\mathrm{X}(\mathrm{BX})$ distribution. We adopted this method by assuming a random sample from a population $T_{1}, T_{2}, \ldots, T_{n}$, from BKBX model with six parameters $\theta=(\nu, \kappa, \varphi, \psi, \vartheta, \tau)$ and KBX model parameters, $\theta_{2}=(\varphi, \psi, \vartheta, \tau)$. We denote $T_{r: n}$ as the $r^{t h}$ order statistics, given the PDF of $T_{r: n}$ can be express as:

$$
f_{r: n}(t ; \theta)=\frac{n !}{(r-1) !(n-r) !} f^{B K B X}(t ; \theta) F^{B K B X}(t ; \theta)^{(r-1)}\left[1-F^{B K B X}(t ; \theta)\right]^{\kappa-r},
$$

Therefore, equation (30) reduces to this form by law of indices,

$$
\begin{aligned}
= & \frac{n !}{(r-1) !(n-r) !} \sum_{k=0}^{\kappa-r}(-1)^{j}\left(\begin{array}{c}
\kappa-r \\
k
\end{array}\right) f^{B K B X}(t ; \theta) \\
& \times \sum_{l=0}^{j+\kappa-1} \eta_{l}\left[F^{B K B X}(t ; \theta)\right]^{l} \sum_{k=0}^{\infty} \mu_{k}\left[\left(F^{B K B X}(t ; \theta)\right)^{k}\right]^{j+r-1}
\end{aligned}
$$


By adopting the binomial expansion by [16], the PDF and CDF of the BKBX distribution. We obtained the PDF of the $r^{t h}$ order statistics of is as follows:

$$
\begin{aligned}
f_{r: n}(t ; \theta)= & \frac{n !}{(r-1) !(n-r) !} \sum_{k=0}^{\kappa-r}(-1)^{k}\left(\begin{array}{c}
\kappa-r \\
k
\end{array}\right)\left(f^{K B X}\left(t ; \theta_{2}\right) \sum_{j=0}^{k+\kappa-1} \eta_{l}\left[F^{K B X}\left(t ; \theta_{2}\right)\right]^{j}\right) \\
& \left.\times\left(\sum_{k=0}^{\infty} d_{j+r-1}\left[F^{K B X}\left(t ; \theta_{2}\right)\right]^{k}\right]\right), \\
= & \frac{n !}{(r-1) !(n-r) !} \sum_{k=0}^{\kappa-r}(-1)^{k}\left(\begin{array}{c}
\kappa-r \\
k
\end{array}\right)\left(f^{K B X}\left(t ; \theta_{2}\right)\right. \\
& \left.\times \sum_{j=0}^{k+\kappa-1} \sum_{k=0}^{\infty} \eta_{l} d_{j+r-1}\left[F^{K B X}\left(t ; \theta_{2}\right)\right]^{k+j}\right), \\
= & f^{K B X}\left(t ; \theta_{2}\right) \sum_{y=0}^{k+j} \frac{\xi_{y}}{y+1} \frac{d}{d t}\left[S^{K B X}\left(t ; \theta_{2}\right)\right]^{y+1},
\end{aligned}
$$

Hence, we obtained the $r^{\text {th }}$ order statistics of the BKBX distribution as:

$$
\begin{aligned}
f_{r: n}(t ; \theta)= & \sum_{y=0}^{k+j} \dot{\xi}_{y} f^{K B X}\left(t ; \theta_{2}(y+1)\right), \\
\dot{\xi}_{y} & =\sum_{j=0}^{k+\kappa-1} \sum_{k=0}^{\infty} \frac{(-1)^{y+1} \gamma_{j, k}}{y+1}\left(\begin{array}{c}
k+j \\
y
\end{array}\right), \\
\xi_{y} & =\dot{\xi}_{y}(y+1), \\
& \gamma_{j, k}=\frac{n !}{(r-1) !(n-r) !} \sum_{k=0}^{\kappa-r}(-1)^{k}\left(\begin{array}{c}
\kappa-r \\
k
\end{array}\right) \eta_{l} d_{j+r-1, k} .
\end{aligned}
$$

\subsection{Rényi Entropy}

An entropy for a given random variable is called the measure of uncertainty used in many areas of research like: applied statistics and reliability in engineering. In the recent years, [33], modified and derived the general formula which was originally proposed by [34], over 5 decades ago. Moreover, [24], provides the expression of this entropies for models with more than one variates. It is given by definition:

$$
I_{R}(\epsilon)=(1-\epsilon)^{-1} \ln \left(\int_{-\infty}^{\infty} f(t)^{\epsilon} d t\right) .
$$

\subsection{Rényi Entropy}

An entropy for a given random variable is called the measure of uncertainty used in many areas of research like: applied statistics and reliability in engineering. In the recent years, [24], modified and derived the general formula which 
was originally proposed by [17], over 5 decades ago. Moreover, [16], provides the expression of this entropies for models with more than one variates. It is given by definition:

$$
I_{R}(\epsilon)=(1-\epsilon)^{-1} \ln \left(\int_{-\infty}^{\infty} f(t)^{\epsilon} d t\right) .
$$

Entropies are measures which quantified the randomness or diversity of a random variable $\mathrm{X}$. They express the expected information uncertainty or content of a PDF. Entropy is a measure that provide important tools to analyze evolutionary processes over time (technical change) and to indicate the variety in the distributions at a particular moment of time. The large value of the Rényi entropy indicates that there is greater uncertainty in the data. Where $\epsilon>0$ and $\epsilon \neq 1$. Using binomial expansion also provided by [16], we can expressed it by denoting:

$$
M_{i}=1-e^{-\left(\tau t_{i}\right)^{2}}, \quad R_{i}=\left[1-\left(M_{i}\right)^{\vartheta \varphi}\right] .
$$

The Rényi entropy for BKBX distribution can be found from equation (13), above as follows:

$$
\begin{aligned}
& \int_{0}^{\infty} f(t)^{\epsilon} d t=\left(\frac{2 \varphi \psi \vartheta \tau^{2}}{B(\nu, \kappa)}\right)^{\epsilon} \int_{0}^{\infty}\left[t_{i} e^{-\left(\tau t_{i}\right)^{2}} M_{i}^{(\vartheta \varphi-1)}\left[1-R_{i}\right]^{\psi \kappa-1}\right]^{\epsilon} \\
& \times\left[1-R_{i}^{\psi}\right]^{\epsilon(\nu-1)} d t . \\
& =\left(\frac{2 \varphi \psi \vartheta \tau^{2}}{B(\nu, \kappa)}\right) \sum_{i=0}^{\epsilon}\left(\begin{array}{c}
\epsilon(\nu-1) \\
i
\end{array}\right)(-1)^{i} \int_{0}^{\infty} t_{i}^{\epsilon} e^{-\epsilon\left(\tau t_{i}\right)^{2}}\left[M_{i}^{(\vartheta \varphi-1)}\right]^{\epsilon} \\
& \times\left[1-R_{i}\right]^{\epsilon(\psi \kappa-1)}\left[1-R_{i}\right]^{\psi} d t . \\
& =\left(\frac{2 \varphi \psi \vartheta \tau^{2}}{B(\nu, \kappa)}\right) \sum_{i=0}^{\epsilon}\left(\begin{array}{c}
\epsilon(\nu-1) \\
i
\end{array}\right)(-1)^{i} \int_{0}^{\infty} t_{i}^{\epsilon} e^{-\epsilon\left(\tau t_{i}\right)^{2}}\left[M_{i}^{(\vartheta \varphi-1)}\right]^{\epsilon} \\
& \times\left[1-R_{i}\right]^{\epsilon(\psi \kappa-1)+\psi_{i}} d t . \\
& =\left(\frac{2 \varphi \psi \vartheta \tau^{2}}{B(\nu, \kappa)}\right) \sum_{i, j=0}^{(\nu-1) \epsilon}\left(\begin{array}{c}
\epsilon(\nu-1) \\
j
\end{array}\right)\left(\begin{array}{c}
\epsilon(\psi \kappa-1)+\psi_{i} \\
i
\end{array}\right)(-1)^{i+j} \\
& \times \int_{0}^{\infty} t_{i}^{\epsilon} e^{-\epsilon\left(\tau t_{i}\right)^{2}}\left[M_{i}^{(\vartheta \varphi-1)}\right]^{\epsilon} d t
\end{aligned}
$$


Therefore, we obtained the Rényi entropy by substituting equation (34), in equation (33) as follows:

$$
\begin{aligned}
I_{R}(\epsilon)= & (1-\epsilon)^{-1} \ln \left\{\left(\frac{2 \varphi \psi \vartheta \tau^{2}}{B(\nu, \kappa)}\right)^{\epsilon}\right. \\
& \times \sum_{i, j=0}^{(\nu-1) \epsilon}\left(\begin{array}{c}
\epsilon(\nu-1) \\
j
\end{array}\right)\left(\begin{array}{c}
\epsilon(\psi \kappa-1)+\psi_{i} \\
i
\end{array}\right)(-1)^{i+j} \\
& \left.\times \int_{0}^{\infty} t_{i}^{\epsilon} e^{-\epsilon\left(\tau t_{i}\right)^{2}}\left[M_{i}^{(\vartheta \varphi-1)}\right]^{\epsilon} d t\right\} .
\end{aligned}
$$

Thus, the Rényi entropy is,

$$
\begin{aligned}
I_{R}(\epsilon)= & (1-\epsilon)^{-1} \ln \left(\frac{2 \varphi \psi \vartheta}{B(\nu, \kappa)}\right)^{\epsilon}+\ln (2 \tau) \\
& +\frac{1}{1-\epsilon} \ln \left\{\sum_{i, j=0}^{(\nu-1) \epsilon}\left(\begin{array}{c}
\epsilon(\nu-1) \\
j
\end{array}\right)\right. \\
& \times\left(\begin{array}{c}
\epsilon(\psi \kappa-1)+\psi_{i} \\
i
\end{array}\right)(-1)^{i+j} \\
& \left.\times \int_{0}^{\infty} t_{i}^{\epsilon} e^{-\epsilon\left(\tau t_{i}\right)^{2}}\left[M_{i}^{(\vartheta \varphi-1)}\right]^{\epsilon} d t\right\} .
\end{aligned}
$$

\subsection{Quantile Function}

Let $Q_{\nu, \kappa}(u)$ be the beta quantile function with parameters $\nu$ and $\kappa$. The quantile function of the BKBX model with $\theta=(\nu, \kappa, \varphi, \psi, \vartheta, \tau)$ parameters, let $t=Q(u)$, using the cumulative distribution function given as:

$$
\begin{aligned}
F^{B K B X}(t ; \theta) & =I_{1-\left\{\left(1-e^{-(\tau t)^{2}}\right)^{\vartheta} \varphi\right\}^{\psi}}(\nu, \kappa) . \\
\text { Let }, u & =I_{1-\left\{\left(1-e^{-(\tau t)^{2}}\right)^{\vartheta} \varphi\right\}^{\psi}}(\nu, \kappa) \\
I_{u}^{-1}(\nu, \kappa) & =1-\left\{\left(1-e^{-(\tau t)^{2}}\right)^{\vartheta \varphi}\right\}^{\psi},
\end{aligned}
$$

multiply both powers by " $1 / \psi$ " to deduce the equation:

$$
\begin{aligned}
& \left(I_{u}^{-1}(\nu, \kappa)\right)^{1 / \psi}=1-\left\{\left\{\left(1-e^{-(\tau t)^{2}}\right)^{\vartheta \varphi}\right\}^{\psi}\right\}^{1 / \psi}, \\
& \left(I_{u}^{-1}(\nu, \kappa)\right)^{1 / \psi}=1-\left\{\left(1-e^{-(\tau t)^{2}}\right)^{\vartheta \varphi}\right\} .
\end{aligned}
$$

Similarly, we again multiply both powers by " $1 / \vartheta \varphi$ " to deduce the equation:

$$
\begin{aligned}
{\left[1-\left(I_{u}^{-1}(\nu, \kappa)\right)^{1 / \psi}\right]^{1 / \vartheta \varphi} } & =\left[\left(1-e^{-(\tau t)^{2}}\right)^{\vartheta \varphi}\right]^{1 / \vartheta \varphi}, \\
{\left[1-\left(1-I_{u}^{-1}(\nu, \kappa)\right)^{1 / \psi}\right]^{1 / \vartheta \varphi} } & =-e^{-(\tau t)^{2}} .
\end{aligned}
$$


Take a logarithm and multiply for both sides by -1 we obtained:

$$
-\ln \left[1-\left(1-I_{u}^{-1}(\nu, \kappa)\right)^{1 / \psi}\right]^{1 / \vartheta \varphi}=(\tau t)^{2},
$$

by taking the square root of both sides:

$$
\begin{aligned}
& \sqrt{\left\{-\ln \left[1-\left(1-I_{u}^{-1}(\nu, \kappa)\right)^{1 / \psi}\right]^{1 / \vartheta \varphi}\right\}}=\sqrt{(\tau t)^{2}} \\
& \left\{-\ln \left[1-\left(1-I_{u}^{-1}(\nu, \kappa)\right)^{1 / \psi}\right]^{1 / \vartheta \varphi}\right\}^{1 / 2}=\left[(\tau t)^{2}\right]^{1 / 2} \\
& \left\{-\ln \left[1-\left(1-I_{u}^{-1}(\nu, \kappa)\right)^{1 / \psi}\right]^{1 / \vartheta \varphi}\right\}^{1 / 2}=\tau t .
\end{aligned}
$$

Therefore, the quantile function of BKBX distribution is given below by taking the inverse making " $t$ " the subject of the formula.

$$
\begin{aligned}
t= & \frac{1}{\tau} \times\left\{-\ln \left[1-\left(1-I_{u}^{-1}(\nu, \kappa)\right)^{1 / \psi}\right]^{1 / \vartheta \varphi}\right\}^{1 / 2}, \\
& \text { where, } \mathrm{t}=\mathrm{Q}_{(\mathrm{u})}, \\
Q_{(u)}= & \frac{1}{\tau} \times\left\{-\ln \left[1-\left(Q_{\nu, \kappa}(u)\right)^{1 / \psi}\right]^{1 / \vartheta \varphi}\right\}^{1 / 2} . \\
& \text { Where, } \mathrm{Q}_{\nu, \kappa}(\mathrm{u})=1-\mathrm{I}_{\mathrm{u}}^{-1}(\nu, \kappa) .
\end{aligned}
$$

\subsection{Skewness and Kurtosis}

Skewness: is a measure of symmetry, or more precisely, the lack of symmetry. A distribution, or data set, is symmetric if it looks the same to the left and right of the center point. The Bowley skewness by [25], is actually a statistical procedure to find the positive or negative skewed distribution on based on different kinds of data depending on area and the suitability of the functions. It is the most popular tool procedure in finding a skewness fit given by.

$$
\begin{aligned}
& S K=\frac{Q_{(3 / 4)}+Q_{(1 / 4)}-2 Q_{(1 / 2)}}{Q_{(3 / 4)}-Q_{(1 / 4)}}, \\
& S K=\frac{Q_{(0.75)}+Q_{(0.25)}-2 Q_{(0.5)}}{Q_{(0.75)}-Q_{(0.25)}} .
\end{aligned}
$$

On the other hand we have, Kurtosis: is a measure of whether the data are heavy-tailed or light-tailed relative to a normal distribution. That is, data sets with high kurtosis tend to have heavy tails, or outliers. Data sets with low kurtosis tend to have light tails, or lack of outliers. A uniform distribution would be the extreme case. The Moors Kurtosis by [26], can be calculated by using the formula given below:

$$
\begin{aligned}
& M K=\frac{Q_{(1 / 8)}+Q_{(3 / 8)}+Q_{(7 / 8)}-Q_{(5 / 8)}}{Q_{(3 / 4)}-Q_{(1 / 4)}}, \\
& M K=\frac{Q_{(0.125)}+Q_{(0.375)}+Q_{(0.875)}-Q_{(0.625)}}{Q_{(0.75)}-Q_{(0.25)}} .
\end{aligned}
$$


B.S.

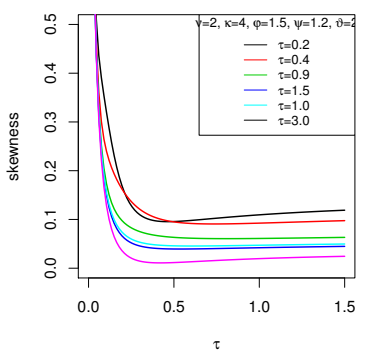

(a) Skewness Shape 1.
B.S.

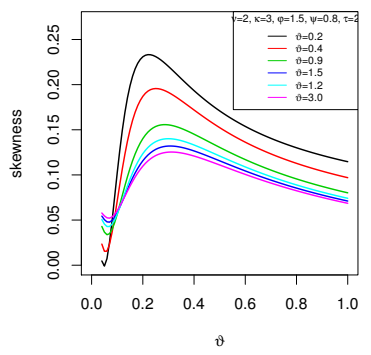

(b) Skewness Shape 2.

Figure 8: Plot of the BKBX Skewness for different parameter values of $\nu, \kappa, \varphi, \psi, \vartheta$ and $\tau$.

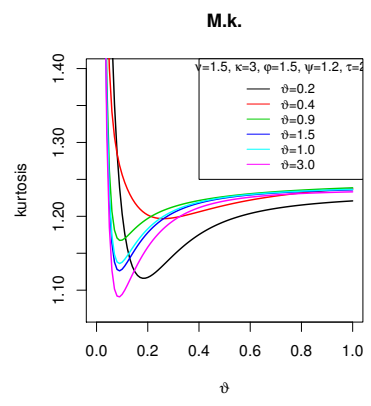

(a) Kurtosis Shape 1.

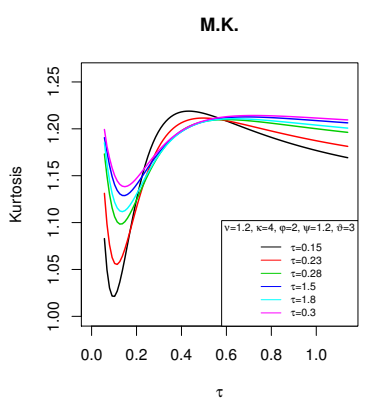

(b) Kurtosis Shape 2 .

Figure 9: Plot of the BKBX Kurtosis for different parameter values of $\nu, \kappa, \varphi, \psi, \vartheta$ and $\tau$. 
Figure (8), shows measure of relative symmetry as normally zero indicates symmetry. The larger its absolute value the more asymmetric the distribution. The positive parameters values indicates a long right tail skeweness.

Figure (9), shows the measure of the relative peakedness of a distribution. For a normal bellshaped distribution (mesokurtic) and a platykurtic distribution (flatter than a normal distribution with shorter tails). Also a leptokurtic distribution (more peaked than a normal distribution with longer tails) respectively.

\subsection{Inference of Estimation:}

The most widely used method for the estimation of parameters of distribution is the maximum likelihood estimation method (MLE) and the moment method. We employ MLE to estimate the unknown parameter of BKBX distribution. The BKBX model with six parameters: $\theta=(\nu, \kappa, \varphi, \psi, \vartheta, \tau)$, given the likelihood function as:

$$
\begin{aligned}
L(\theta)= & \prod_{i=0}^{n}\left\{\frac{2}{B(\nu, \kappa)} \varphi \psi \vartheta \tau^{2} t e^{-(\tau t)^{2}}\left\{1-e^{-(\tau t)^{2}}\right\}^{(\vartheta \varphi-1)}\right. \\
& \times\left[1-\left\{1-e^{\left.-(\tau t)^{2}\right)^{\vartheta \varphi}}\right\}\right]^{\psi \kappa-1} \\
& \left.\times\left[1-\left[1-\left\{\left(1-e^{-(\tau t)^{2}}\right)^{\vartheta \varphi}\right\}\right]^{\psi}\right]^{\nu-1}\right\}
\end{aligned}
$$

for $\nu>0, \kappa>0, \varphi>0, \psi>0, \vartheta>0, \tau>0$. Let $t=\left(t_{1}, t_{2}, . ., t_{n}\right)^{T}$ be a random sample of size "n" from BKBX with parameters: $\theta=(\nu, \kappa, \varphi, \psi, \vartheta, \tau)^{T}$, for $\mathrm{i}=1,2, \ldots, \mathrm{n}$. Then the log-likelihood function for is given by:

$$
\begin{aligned}
\ell(\theta)= & n \ln \left\{\frac{2 \varphi \psi \vartheta}{B(\nu, \kappa)}\right\}+2 \sum_{i=1}^{n} \ln \left(\tau t_{i}\right)-\sum_{i=1}^{n}\left(\tau t_{i}\right)^{2} \\
& +(\vartheta \varphi-1) \sum_{i=1}^{n} \ln \left[1-e^{-\left(\tau t_{i}\right)^{2}}\right] \\
& +(\psi \kappa-1) \sum_{i=1}^{n} \ln \left[1-\left(1-e^{-\left(\tau t_{i}\right)^{2}}\right)^{\vartheta \varphi}\right] \\
& +(\nu-1) \sum_{i=1}^{n} \ln \left[1-\left[1-\left(1-e^{-(\tau t)^{2}}\right)^{\vartheta \varphi}\right]^{\psi}\right] .
\end{aligned}
$$

Equation (47), reduces to as follows:

$$
\begin{aligned}
\ell(\theta)= & n(\ln (2)+\ln (\varphi)+\ln (\psi)+\ln (\vartheta))+2 n \ln (\tau) \\
& +\sum_{i=1}^{n} \ln \left(t_{i}\right)-\sum_{i=1}^{n}\left(D_{i}\right)^{2}-n \ln [B(\nu, \kappa)]+(\vartheta \varphi-1) \sum_{i=1}^{n} \ln \left(M_{i}\right) \\
& +(\psi \kappa-1) \sum_{i=1}^{n} \ln \left(R_{i}\right)+(\nu-1) \sum_{i=1}^{n} \ln \left\{1-\left(R_{i}\right)^{\psi}\right\} .
\end{aligned}
$$


By applying the partial derivatives of the equation (47), that is, the log-likelihood of the BKBX new model above with respect to $\theta=(\nu, \kappa, \varphi, \psi, \vartheta, \tau)$ components of the score vectors: $U_{\Phi}=\left(U_{\nu}, U_{\kappa}, U_{\varphi}, U_{\psi}, U_{\vartheta}, U_{\tau}\right)$ and by denoting:

$$
\begin{aligned}
D_{i} & =\tau t_{i} \\
K_{i} & =e^{-\left(D_{i}\right)^{2}} \\
M_{i} & =1-e^{-\left(K_{i}\right)^{2}} \\
R_{i} & =1-\left(M_{i}\right)^{\vartheta \varphi}
\end{aligned}
$$

were obtained as follows:

$$
\begin{aligned}
& \frac{\partial l}{\partial \nu}=U_{\nu}=-n \Psi(\nu)+n \Psi(\nu+\kappa)+\sum_{i=1}^{n} \ln \left[1-\left(R_{i}\right)^{\psi}\right]=0 \\
& \frac{\partial l}{\partial \kappa}=U_{\kappa}=-n \Psi(\kappa)+n \Psi(\nu+\kappa)+\psi \sum_{i=1}^{n} \ln \left(R_{i}\right)=0 \\
& \frac{\partial l}{\partial \varphi}=U_{\varphi}=\frac{n}{\varphi}+\vartheta \sum_{i=1}^{n} \ln \left(M_{i}\right)+(1-\psi \kappa) \sum_{i=1}^{n} \frac{\left(M_{i}\right)^{\vartheta \varphi} \ln M_{i}}{R_{i}} \\
& +(\nu-1) \sum_{i=1}^{n} \frac{\left[\psi\left[R_{i}\right]^{\psi-1}\left(M_{i}\right)^{\vartheta \varphi} \ln \left(M_{i}\right)\right]}{1-\left(R_{i}\right)^{\psi}}=0 . \\
& \frac{\partial l}{\partial \psi}=U_{\psi}=\frac{n}{\psi}+\kappa \sum_{i=1}^{n} \ln R_{i}+(1-\nu) \sum_{i=1}^{n} \frac{\left(R_{i}\right)^{\psi} \ln R_{i}}{1-\left(R_{i}\right)^{\psi}}=0 . \\
& \frac{\partial l}{\partial \vartheta}=U_{\vartheta}=\frac{n}{\vartheta}+\varphi \sum_{i=1}^{n} \ln \left(M_{i}\right)+(1-\psi \kappa) \sum_{i=1}^{n} \frac{\left(M_{i}\right)^{\vartheta \varphi} \ln M_{i}}{R_{i}} \\
& +(\nu-1) \sum_{i=1}^{n} \frac{\left[\psi\left[R_{i}\right]^{\psi-1}\left(M_{i}\right)^{\vartheta \varphi} \ln \left(M_{i}\right)\right]}{1-\left(R_{i}\right)^{\psi}}=0 . \\
& \frac{\partial l}{\partial \tau}=\frac{2 n}{\tau}+\frac{2}{\tau} \sum_{i=1}^{n}\left(K_{i}\right)^{2}+(\vartheta \varphi-1) \sum_{i=1}^{n} \frac{\tau^{2} M_{i} K_{i}^{2}}{M_{i}} \\
& +(1-\psi \kappa) \sum_{i=1}^{n} \frac{2 \varphi \psi\left(1-R_{i}\right)^{\psi} M_{i}\left(R_{i}\right)^{\vartheta \varphi-1}}{\left.\left(R_{i}\right)\right)^{\psi}} \\
& +2 \varphi \vartheta \tau(\nu-1) \sum_{i=1}^{n} \frac{\left(D_{i}\right)^{2} M_{i}\left(R_{i}\right)^{\vartheta \varphi-1}}{1-\left[\left(R_{i}\right)\right)^{\psi}}=0 .
\end{aligned}
$$

Where $\omega($.$) represents the digamma function obtaining the estimations of the$ score functions in equations: (48), (49), (50), (51), (52) and (53), using the Maple software to simplify the solution iteratively. The provision of interval 
estimation for the hypothesis test based on the new model unknown parameters, an information matrix of all the BKBX distribution where $\varrho<0$, and also the $6 \times 6$ matrix with $I_{n}=I_{n}(\theta)(\theta=(\nu, \kappa, \varphi, \psi, \vartheta, \tau))$ is written as follows:

$$
I_{n}=\left[\begin{array}{llllll}
I_{\nu \nu} & I_{\nu \kappa} & I_{\nu \varphi} & I_{\nu \psi} & I_{\nu \vartheta} & I_{\nu \tau} \\
I_{\kappa \nu} & I_{\kappa \kappa} & I_{\kappa \varphi} & I_{\kappa \psi} & I_{\kappa \vartheta} & I_{\kappa \tau} \\
I_{\varphi \nu} & I_{\varphi \kappa} & I_{\varphi \varphi} & I_{\varphi \psi} & I_{\varphi \vartheta} & I_{\varphi \tau} \\
I_{\psi \nu} & I_{\psi \kappa} & I_{\psi \varphi} & I_{\psi \psi} & I_{\psi \vartheta} & I_{\psi \tau} \\
I_{\vartheta \nu} & I_{\vartheta \kappa} & I_{\vartheta \varphi} & I_{\vartheta \psi} & I_{\vartheta \vartheta} & I_{\vartheta \tau} \\
I_{\tau \nu} & I_{\tau \kappa} & I_{\tau \varphi} & I_{\tau \psi} & I_{\tau \vartheta} & I_{\tau \tau}
\end{array}\right]
$$

\section{Simulation Studies}

We conduct a Monte Carlo simulation using the inverse CDF method considering the quantile function of the new BKBX model from Equation (43), used to generate the random numbers for the simulation results and also compare with any existing model for validating the efficiency of developing the new model.

\subsection{Monte Carlo Inverse CDF method}

In this part we employ an algorithm that generate a sample of size "n" randomly from the $E T-E(E T-E(\theta)$ model for the parameters $(\theta=\nu, \kappa, \varphi, \psi, \vartheta, \tau)$ and the sample size " $n "$. The simulation process has the following steps below:

1. Step 1. We set the sample size "n" and $\theta$.

2. Step 2. We set the initial value $t_{o}$ for a random procedure.

3. Step 3. We set $j=1$.

4. Step 4. We generate $U \sim U$ niform $(0,1)$.

5. Step 5. We set an Update $t_{o}$ by using the Monte Carlo simulation process.

6. Step 6. If $-t_{o}-t \mid \leq \epsilon$, (moderately small, $\epsilon>0$ tolerance limit). Then, $t *$ to the desired sample from $F(t)$.

7. Step 7. If $-t_{o}-t \mid>$, then, set $t_{o}=t *$ and then proceed to step 5 .

8. Step 8. We repeat the procedure steps from $4-7$, for $j=1,2, \ldots, n$ and obtained $t_{1}, t_{2}, \ldots, t n$.

A simulation studies was done to access and validate the efficiency of the new model as we adopt the Monte Carlo simulation using the inverse CDF method of sampling considering the quantile function above of the new model which we study to access the performance of the MLE's of $\theta=(\nu, \kappa, \varphi, \psi, \vartheta, \tau)$ parameters.

We also generate different "n" sample observation from Equation (43). The parameter estimation was done by maximum likelihood method. We considered different sample size $n=(50,150,300,500)$ and the number of repetition is 1000 respectively. The true parameters value at different sets for each model were used respectively. 
Table (1) and Table (2), shows that the mean, bias and MSE of the estimate parameters. We observed that, when we increase sample size "n", the mean is converging towards the true-value while, the bias and MSE are becoming very smaller close to zero for both BKBX and BWB models. Likewise, the blue colour estimated values indicates how the bias and MSE are decreasing with increase of the sample sizes $\mathrm{n}=(50,150,300,500)$ respectively.

Table 1: The mean, bias and MSE on Monte Carlo simulation for BKBX parameters values $\theta=(2,2,0.6,0.4,2,2)$ and BWB parameter values $\theta=(2,2,0.4,2)$.

\begin{tabular}{|c|c|c|c|c|c|c|}
\hline Parameter & True value & Sample size " $\mathrm{n}$ " & Mean & Bias & MSE & (AIC) \\
\hline \multicolumn{7}{|l|}{ BKBX } \\
\hline \multirow{3}{*}{$\nu$} & \multirow{3}{*}{2} & 50 & 2.1822 & 0.1822 & 0.0453 & \\
\hline & & & & & & \\
\hline & & $\begin{array}{l}300 \\
500\end{array}$ & $\begin{array}{l}2.1240 \\
2.1247\end{array}$ & $\begin{array}{l}0.1247 \\
0.1247\end{array}$ & 0.0164 & \\
\hline \multirow[b]{3}{*}{$\kappa$} & \multirow[b]{3}{*}{2} & 50 & 2.6415 & 0.6415 & 0.4242 & \\
\hline & & 150 & 2.6283 & 0.6283 & 0.4003 & \\
\hline & & 300 & 2.6263 & & & \\
\hline \multirow{4}{*}{$\varphi$} & \multirow{4}{*}{0.6} & & & & & \\
\hline & & 150 & 0.6134 & 0.0134 & 0.3487 & \\
\hline & & 300 & & & 0.3401 & \\
\hline & & 500 & 0.6085 & 0.0085 & 0.2909 & (106) \\
\hline \multirow[b]{3}{*}{$\psi$} & \multirow[b]{3}{*}{0.4} & 50 & 0.5261 & 0.1261 & 0.0473 & \\
\hline & & 150 & 0.5066 & 0.1066 & 0.0344 & \\
\hline & & 300 & 0.4784 & 0.0784 & 0.0303 & \\
\hline \multirow{4}{*}{$\vartheta$} & \multirow{4}{*}{2} & & & & & \\
\hline & & $\begin{array}{l}50 \\
150\end{array}$ & 2.1576 & 0.1576 & 0.0282 & \\
\hline & & 300 & 2.1205 & 0.1205 & 0.0223 & \\
\hline & & 500 & 2.1071 & 0.1071 & 0.0131 & \\
\hline \multirow[b]{3}{*}{$\tau$} & \multirow{4}{*}{2} & 50 & 2.7078 & 0.7078 & 0.0319 & \\
\hline & & 150 & 2.4729 & 0.4729 & 0.0282 & \\
\hline & & 300 & 2.2058 & 0.2058 & 0.0207 & \\
\hline & & & 2.1046 & 0.1046 & & \\
\hline \multirow{2}{*}{\multicolumn{2}{|c|}{ BWB }} & & & & & \\
\hline & & 50 & 2.0286 & 0.0286 & 0.2886 & \\
\hline \multirow{3}{*}{$\nu$} & \multirow{3}{*}{2} & 150 & 2.0538 & 0.0538 & 0.1314 & \\
\hline & & 300 & 2.0436 & 0.0436 & 0.0716 & \\
\hline & & & 2.0394 & 0.0394 & 0.0440 & \\
\hline \multirow{4}{*}{$\kappa$} & \multirow{4}{*}{2} & 50 & 2.0233 & 0.0233 & 0.1840 & \\
\hline & & & 2.0317 & 0.0317 & 0.1027 & \\
\hline & & 300 & 2.0094 & 0.0094 & 0.0435 & \\
\hline & & 500 & 2.0139 & 0.0139 & 0.0284 & (112) \\
\hline \multirow{4}{*}{$\varphi$} & \multirow{4}{*}{0.4} & & & & & \\
\hline & & 150 & 0.4023 & 0.0023 & 0.0021 & \\
\hline & & 300 & 0.3995 & -0.0005 & 0.0011 & \\
\hline & & 500 & 0.3986 & -0.0014 & 0.0007 & \\
\hline \multirow[b]{3}{*}{$\tau$} & \multirow[b]{3}{*}{2} & 50 & 2.1194 & 0.1194 & 0.2284 & \\
\hline & & 150 & 2.0785 & 0.0785 & 0.0930 & \\
\hline & & $\begin{array}{l}300 \\
500\end{array}$ & $\begin{array}{l}2.0785 \\
2.0496\end{array}$ & $\begin{array}{l}0.0785 \\
0.0496\end{array}$ & $\begin{array}{l}0.0846 \\
0.0574\end{array}$ & \\
\hline
\end{tabular}


Table 2: The Bias and RMSE on Monte Carlo simulation for parameters values $\theta=4,4,1,1,4,4$ BWB parameter values $\theta$

\begin{tabular}{|c|c|c|c|c|c|c|}
\hline $\begin{array}{l}\text { Parameter } \\
\end{array}$ & True value & Sample size " $\mathrm{n}$ " & Mean & Bias & $\overline{\text { MSE }}$ & (AIC) \\
\hline \multicolumn{7}{|l|}{ BKBX } \\
\hline \multirow{4}{*}{$\nu$} & \multirow{4}{*}{4} & 50 & 4.6421 & 0.6421 & 0.4448 & \\
\hline & & 150 & 4.5841 & 0.5841 & 0.3621 & \\
\hline & & 300 & 4.5497 & 0.5497 & 0.3166 & \\
\hline & & & & & 0.2934 & \\
\hline \multirow{4}{*}{$\kappa$} & \multirow{4}{*}{4} & 50 & 4.7252 & 0.7252 & 0.6222 & \\
\hline & & 150 & 4.6681 & 0.6681 & 0.5273 & \\
\hline & & 300 & & & 0.3949 & \\
\hline & & & 4.4889 & 0.4889 & 0.3446 & \\
\hline \multirow[b]{3}{*}{$\varphi$} & \multirow[b]{3}{*}{1} & 50 & 1.0863 & 0.0863 & 0.1037 & \\
\hline & & 150 & 1.0578 & 0.0578 & 0.0935 & \\
\hline & & $\begin{array}{l}300 \\
500\end{array}$ & $\begin{array}{l}1.0397 \\
\end{array}$ & 0.0397 & 0.0361 & \\
\hline \multirow{4}{*}{$\psi$} & \multirow{4}{*}{1} & & & & & \\
\hline & & $\begin{array}{l}150 \\
150\end{array}$ & $\begin{array}{l}1.07906 \\
1.0660\end{array}$ & $\begin{array}{l}0.0660 \\
0.060\end{array}$ & 0.0228 & \\
\hline & & 300 & & & 0.0211 & \\
\hline & & 500 & 1.0523 & 0.0523 & 0.0163 & \\
\hline \multirow{4}{*}{$\vartheta$} & \multirow{4}{*}{4} & 50 & 4.1024 & 0.1024 & 0.5346 & \\
\hline & & 150 & 4.0843 & 0.0843 & 0.4525 & \\
\hline & & 300 & 4.0615 & 0.0615 & 0.4173 & \\
\hline & & 500 & 4.0342 & 0.0342 & 0.3101 & \\
\hline \multirow{4}{*}{$\tau$} & \multirow{4}{*}{4} & 50 & & 0.4565 & 0.3836 & \\
\hline & & 150 & 4.4251 & 0.4251 & 0.3576 & \\
\hline & & 300 & 4.4102 & 0.1302 & 0.3303 & \\
\hline & & 500 & 4.4039 & 0.4039 & 0.2951 & \\
\hline \multirow[t]{2}{*}{ BWB } & \multirow{5}{*}{4} & & & & & \\
\hline & & 50 & 4.0535 & 0.0535 & 0.3878 & \\
\hline & & & 4.1326 & 0.1326 & & \\
\hline$\nu$ & & 300 & 4.0986 & 0.0986 & 0.0959 & \\
\hline & & 500 & 4.1073 & 0.1073 & 0.0977 & \\
\hline \multirow{4}{*}{$\kappa$} & \multirow{4}{*}{4} & 50 & 4.1419 & 0.1419 & 0.1551 & \\
\hline & & 150 & 4.1340 & 0.1340 & 0.1050 & \\
\hline & & $\begin{array}{l}300 \\
500\end{array}$ & 4.1073 & 0.1073 & 0.0511 & \\
\hline & & & & & & (109) \\
\hline \multirow{4}{*}{$\varphi$} & \multirow{4}{*}{1} & 50 & 1.0352 & 0.0352 & 0.0239 & \\
\hline & & 150 & & -0.0046 & 0.0071 & \\
\hline & & 300 & 0.9931 & -0.0069 & 0.0039 & \\
\hline & & 500 & 0.9908 & -0.0092 & 0.0025 & \\
\hline \multirow{3}{*}{$\tau$} & \multirow[b]{3}{*}{4} & 50 & 4.0047 & 0.0047 & 0.0678 & \\
\hline & & $\begin{array}{l}150 \\
300\end{array}$ & 3.9990 & -0.0010 & 0.2140 & \\
\hline & & $\begin{array}{l}300 \\
500\end{array}$ & $\begin{array}{l}3.9967 \\
3.9839 \\
\end{array}$ & $\begin{array}{l}-0.0033 \\
-0.0161\end{array}$ & $\begin{array}{l}0.0164 \\
0.0280\end{array}$ & \\
\hline
\end{tabular}

\section{Application of Real Datasets:}

Here, we apply data set to clarify the fitness of Beta Kumaraswamy-Burr Type $\mathrm{X}$ distribution is a better model than the baselines, Burr type $\mathrm{X}$ and Kumaraswamy-Burr Type X distributions respectively.

\subsection{Life of Fatigue Fracture of Kevlar Dataset:}

"This data set consists of 306 observations of the life of fatigue fracture of Kevlar dataset, originally obtained by workers at the UK National Physical Laboratory. On the other side they fail to include the units of measurements in the work". The data have also been analyzed by [27].

\subsection{AIDS Clinical Trials Group Study 320 Dataset:}

"The data come from a double-blind, placebo-controlled trial that compared the three-drug regimen of indinavir (IDV), open label zidovudine (ZDV) or stavudine $(\mathrm{d} 4 \mathrm{~T})$ and lamivudine (3TC) with the two-drug regimen of zidovudine or stavudine and lamivudine in HIV-infected patients" by [28]. 


\subsection{Nicotine Measurements Dataset:}

"The following data is about 346 nicotine measurements made from several brands of cigarettes in 1998. The data have been collected by the Federal Trade Commission which is an independent agency of the US government, whose main mission is the promotion of consumer protection by [29].

\subsection{Criterion:}

The criterion like: log-likelihood, Akaike information criterion, consistent Akaike information criterion and Bayesian information criterion for the data set above so as to compare the models and to check which have least or smaller $-2 \ell$, $A_{k} \mathrm{IC}, A_{k} \mathrm{ICC}, B_{\text {ayes }} \mathrm{IC}, \mathrm{K}-\mathrm{SM}$ and $p-$ Value values. The distribution of the datasets was skewed to the left, right and symmetric respectively.

Table 3: The ML estimates, $-2 \ell$ log-likelihood, $A_{k}$ IC , $A_{k}$ ICC and $B_{\text {ayes }}$ IC, K-SM and $p-$ Value values. for the life of fatigue fracture of Kevlar dataset.

\begin{tabular}{|c|c|c|c|c|c|c|c|c|c|}
\hline Model & MLE & $-2 \ell(\theta)$ & $A_{k}$ IC & $A_{k} \mathrm{ICC}$ & $B_{\text {ayes IC }}$ & K-SM & Probability (p-value) & Cramer-von Mises (CvM) Test & Watson (W) Test \\
\hline Beta Kum-Burr Type X & $\begin{array}{c}\nu=1.3782 \\
\kappa=2.4670 \\
\varphi=6.349 \\
\psi=0.905 \\
\vartheta=1.9045 \\
\tau=3.1353\end{array}$ & 121 & 248 & 256 & 253 & 0.2776 & 0.7841 & 0.1574 & 0.1494 \\
\hline Kum-Burr Type X & $\begin{array}{l}\varphi=1.2519 \\
\psi=0.886 \\
\vartheta=4.646 \\
\tau=3.2238\end{array}$ & 123 & 252 & 259 & 256 & 0.3826 & 0.4324 & 0.2216 & 0.2513 \\
\hline Burr Type X & $\begin{array}{c}y=3.532 \\
\tau=1.0269\end{array}$ & 127 & 255 & 266 & 258 & 0.3772 & 0.4018 & 0.5432 & 0.5285 \\
\hline
\end{tabular}

Table 4: The ML estimates, log-likelihood, $A_{k}$ IC, $A_{k}$ ICC, $B_{\text {ayes }}$ IC for the measurements of the AIDS clinical trials group study 320 dataset.

\begin{tabular}{|c|c|c|c|c|c|c|c|c|c|}
\hline Model & MLE & $-2 \ell(\theta)$ & $A_{k} \mathrm{IC}$ & $A_{k} \mathrm{ICC}$ & $B_{a y e s} \mathrm{IC}$ & K-SM & Probability (p-value) & Cramer-von Mises (CvM) Test & Watson (W) Test \\
\hline Beta Kum-Burr Type X & $\begin{array}{l}\nu=0.0439 \\
\kappa=0.5237 \\
\varphi=0.2158 \\
\psi=0.2467 \\
\vartheta=0.0345 \\
\tau=0.0882\end{array}$ & 220.6718 & 449.3437 & 450.2326 & 456.9918 & 0.3112 & 0.8504 & 0.6214 & 0.1732 \\
\hline Beta-Weibull & $\begin{array}{l}\varphi=0.5237 \\
\psi=0.0847 \\
\vartheta=0.2352 \\
\tau=0.0483\end{array}$ & 238.1199 & 482.2399 & 482.7617 & 487.9760 & 0.2542 & 0.5908 & 0.1845 & 0.1987 \\
\hline Gen. Gompertz & $\begin{array}{l}\varphi=0.0262 \\
\psi=0.0896 \\
\tau=0.0834\end{array}$ & 222.6718 & 450.48819 & 451.0099 & 476.2242 & 0.1832 & 0.2253 & 0.2698 & 0.2309 \\
\hline Gen. Exponential & $\begin{array}{l}\vartheta=0.0845 \\
\tau=0.0834\end{array}$ & 231.7653 & 462.8646 & 464.6096 & 470.1024 & 0.1703 & 0.2145 & 0.3986 & 0.3608 \\
\hline
\end{tabular}

\section{Summary Of Comments On The Simulation Results}

In summary we proposed a new model called Beta Kumaraswamy BurrType $\mathrm{X}(\mathrm{BKBX})$ with six parameters $\theta=(\nu, \kappa, \varphi, \psi, \vartheta, \tau)$ that extends and 


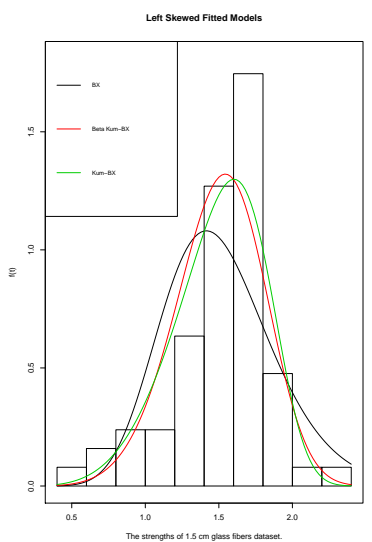

(a) Histogram For Left Skewed Dataset.

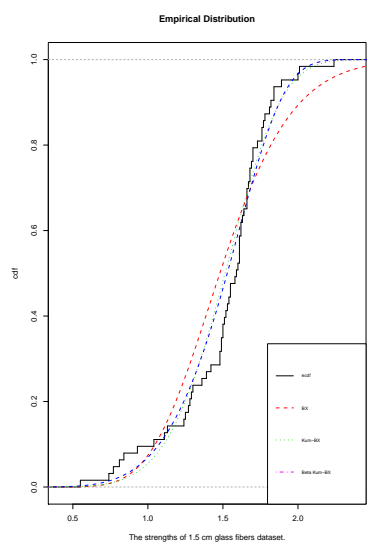

(b) Empirical Distribution 1.

Figure 10: Graph of the histogram and empirical distribution for life of fatigue for Kevlar dataset.

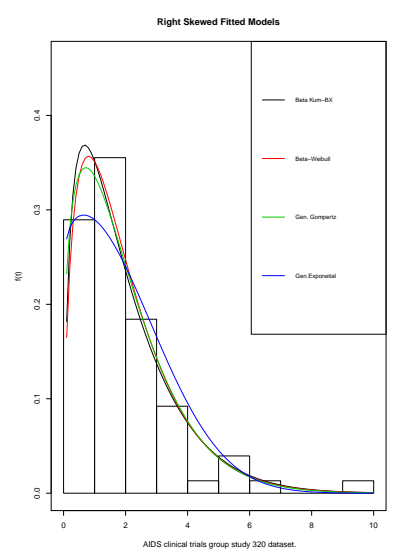

(a) Histogram For Right Skewed Dataset.

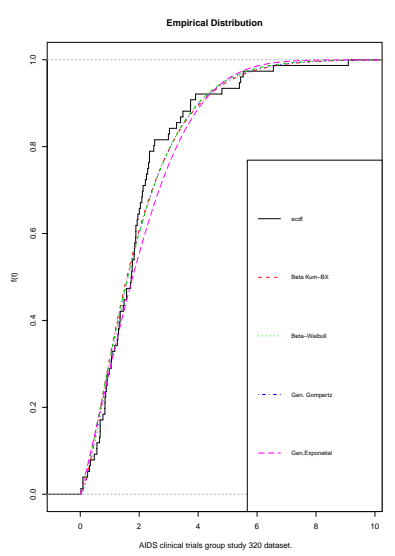

(b) Empirical Distribution 2.

Figure 11: Graph of the histogram and empirical distribution for Beta Kum-BX with existing different models of AIDS clinical trials group study 320 dataset. 
Table 5: The ML estimates, log-likelihood, $A_{k}$ IC, $A_{k}$ ICC, $B_{a y e s}$ IC for Nicotine measurements dataset.

\begin{tabular}{|c|c|c|c|c|c|c|c|c|c|}
\hline Model & MLE & $-2 \ell(\theta)$ & $A_{k} \mathrm{IC}$ & $A_{k}$ ICC & $B_{a y e s} \mathrm{IC}$ & K-SM & probability (p-value) & Cramer-von Mises (CvM) Test & Watson $(W)$ Test \\
\hline Beta Kum BX & $\begin{array}{l}\nu=0.0646 \\
\kappa=0.0357 \\
\varphi=0.0296 \\
\psi=0.0324 \\
\vartheta=0.0428 \\
\tau=0.0475\end{array}$ & 97.0835 & 200.1675 & 201.3892 & 203.5740 & 0.5416 & 0.7245 & 0.1206 & 0.1173 \\
\hline Beta-Weibull & $\begin{array}{l}\varphi=0.0253 \\
\psi=0.0289 \\
\vartheta=0.0834 \\
\tau=0.0243\end{array}$ & 97.0838 & 202.1670 & 201.4307 & 206.7090 & 0.7212 & 0.5304 & 0.2526 & 0.2417 \\
\hline Gen. Gompertz & $\begin{array}{l}\varphi=0.0137 \\
\psi=0.0339 \\
\tau=0.2352\end{array}$ & 99.1344 & 204.2688 & 205.5319 & 207.6752 & 0.5043 & 0.5432 & 0.4187 & 0.3920 \\
\hline Gen. Exponential & $\begin{array}{l}\vartheta=0.0742 \\
\tau=0.0234\end{array}$ & 102.9185 & 209.8371 & 210.4383 & 212.1080 & 0.3490 & 0.2742 & 0.5205 & 0.5124 \\
\hline
\end{tabular}

generalized the Kum-G family, Burr type $\mathrm{X}$ distributions and many sub-models by the family of BK-G family which was proposed by [32].

We obtained the distributional properties like: probability density function, cumulative distribution function, hazard function and their expansions. Also, the statistical properties like: quantile function, Browley skewness, Moors Kurtosis were also obtained. The parameters were estimated by using maximum likelihood estimation (MLE) methods. A simulation study for the model parameters was conducted by given different true values as we can see the higher you increase the " $n$ " bias the smaller the mean square error becomes at each level of the simulation process.

Based on the simulation results for the comparison using the Akaike information criterion (AIC) as to check the best model in-terms of providing a meaningful and exact true value and also how the mean, bias and root mean square errors were obtained.

From Table (1) and Table (2), it was shown that as the sample sizes at each level of the process the bias and the mean square error (MSE) decreases with increasing of the sample sizes, where it proven that the BKBX is the best compare to Beta-Weibull model in-terms of comparison but on the other hand the AIC value of BKBX gives the smallest value of the context that not always the best but rather based on the true values used in the simulation study respectively.

\subsection{Summary Of Comments On The Real Data}

Table 3, 4 and 5 above and below shows MLEs for the individual fitted model

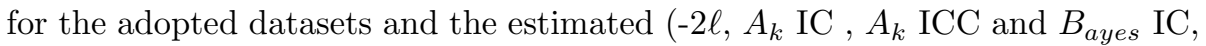
$\mathrm{K}-\mathrm{SM}$ and $p-$ Value), where the results obtained indicates clearly that the Beta Kumaraswamy-Burr Type $\mathrm{X}$ is a stronger with highest peak, flexible and vital to its sub-models and other existing models like: Beta- Weibull, generalized Gompertz and generalized exponential were used here for fitting the data set respectively.

These model as fitted based on the above datasets proves that it is a good example for modeling left, right skewed and symmetric datasets. Also, the 


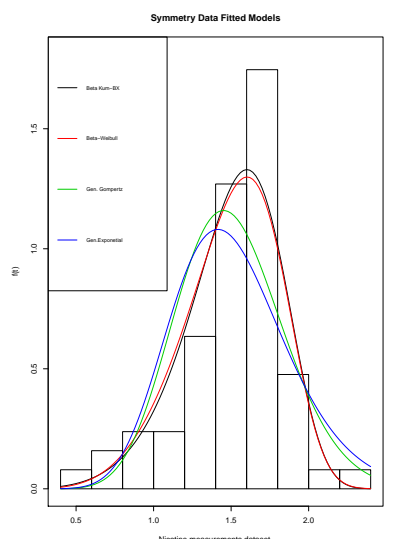

(a) Histogram For Symmetry Dataset.

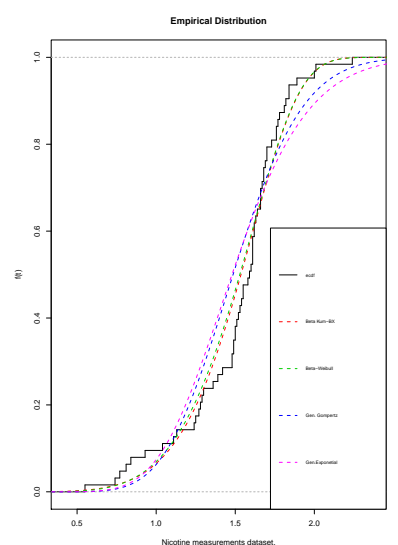

(b) Empirical Distribution 3.

Figure 12: Graph of the histogram and empirical distribution for Beta Kum-BX with existing different models for the Nicotine Measurements dataset.

likelihood ratio test with the hypothesis $H_{o}: a=b=1$ versus $H_{1}: a \neq 1, b \neq 1$. Based on the data above $\omega=20.567>5.991=\chi_{4 ; 0.05}$, we therefore reject the null hypothesis. Figure 10,11 and 12 above and below for the plot of densities which compares the models with the histogram empirical graphs proves that Beta Kum-BX is closer to the histogram peak point than the other models which shows the flexibility of the new model with strong properties. On contrast it shows that based on this three applicable datasets from different areas which fits Beta kumaraswamy Burr type X (Beta Kum-BX) distribution having the

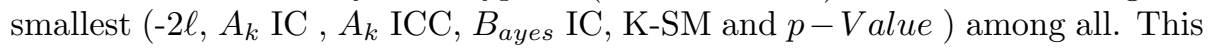
suggest that the Beta Kum-BX distribution is very good in modeling left and right skewed and also some symmetric datasets respectively.

In other to validate the goodness-of-fit for the new model based on the datasets at different level applied like; laboratory dataset, Hiv/Aids (censored) and nicotine measurement for smokers with all comes from science and medical respectively. The used of the test statistics for each model both the new and existing are: Cramer-von Mises (CvM) and the Watson (tests) by [30]. In general view, the suitability of a model based on any datasets apply depends on how smaller the values obtained at each test result for a given datasets where it clearly proves that the Kolmogorov-Smirnov test, $p$ - Value, Cramer-von Mises $(\mathrm{CvM})$ and the Watson (tests) were obtained in all the three provided tables showing the flexibility and efficiency of the new models Beta Kum-BX with six parameters having a better fitness as well as the histogram and empirical graphs respectively.Lastly we hope that the proposed model and its generated models will attract wider applications in several areas among others. 


\section{General Conclusion:}

A new model was introduced called Beta Kumaraswamy Burr type X (Beta Kum-BX) with six parameters $(\nu, \kappa, \vartheta, \psi, \vartheta, \tau)$ that extends the Kum-G family, and Burr type X distributions by the family of Beta Kum-G family which was proposed by [8]. We obtain the distributional properties like: PDF, CDF and shapes of hazard function and their expansions. The unknown parameters were estimated using Bayes and MLE methods apply on three (3) different real data sets on medical (normally fits beta model), hydrological (normally fits $\mathrm{Ku}-$ maraswamy model) and engineering (normally fits Burr type X model) by using the goodness of fit is clarified.

This new model allocate a better fitness, flexibility and vital to use in medical, survival and reliability analysis area when the data follows and suits a Beta Kumaraswamy Burr type X distribution than some its sub models and also different family models. It is a very good model for symmetric, right and left skewed data sets. We recommend medical practitioners, doctors, engineers, statisticians to adopt this suitable Beta Kum-BX in modeling their large group of data as its consist of three strong models property.

\subsection{Acknowledgement}

The authors are very grateful to the Universiti Putra Malaysia for sponsoring the fund together with the referee for suggestions and comments which significantly improved the quality of the manuscript.

\section{References}

[1] Gupta, A.K. and Nadarajah, S. eds., 2004. Handbook of beta distribution and its applications. CRC press.

[2] Kumaraswamy, P., 1980. A generalized probability density function for double-bounded random processes. Journal of Hydrology, 46(1), pp.79-88.

[3] Eugene N., Lee C. and Famoye F., 2002. Beta-normal distribution and its applications. Commun Statist Theor Meth 31: 497-512.

[4] Jones, M.C., 2004. Families of distributions arising from distributions of order statistics. Test, 13(1), pp.1-43.

[5] Jones, M.C., 2009. Kumaraswamy's distribution: A beta-type distribution with some tractability advantages. Statistical Methodology, 6(1), pp.70-81.

[6] Cordeiro G.M. and De Castro M., 2011. A new family of generalized distributions. J Stat Comput Simul 81: 883-893.

[7] Alzaatreh, A., Lee, C. and Famoye, F., 2013. A new method for generating families of continuous distributions. Metron, 71(1), pp.63-79. 
[8] Handique, L. and Chakraborty, S., 2016. Beta generated Kumaraswamy-G and other new families of distributions. arXiv preprint arXiv:1603.00634.

[9] Burr, I.W., 1942. Cumulative frequency functions. The Annals of mathematical statistics, 13(2), pp.215-232.

[10] Raqab, M.Z., 1998. Order statistics from the Burr type X model. Computers and Mathematics with Applications, 36(4), pp.111-120.

[11] Raqab, M.Z. and Kundu, D., 2006. Burr type X distribution: revisited. Journal of Probability and Statistical Sciences, 4(2), pp.179-193.

[12] Surles, J.G. and Padgett, W.J., 2001. Inference for reliability and stressstrength for a scaled Burr Type X distribution. Lifetime Data Analysis, $7(2)$, pp.187-200.

[13] Surles, J.G. and Padgett, W.J., 2005. Some properties of a scaled Burr type X distribution. Journal of Statistical Planning and Inference, 128(1), pp.271-280.

[14] Merovci, F., Khaleel, M.A., Ibrahim, N.A. and Shitan, M., 2016. The beta Burr type X distribution properties with application. SpringerPlus, pp.118.

[15] Khaleel, M.A., Ibrahim, N.A., Shitan, M. and Merovci, F., 2016. Some properties of Gamma Burr type X distribution with Application. AIP Conference proceedings.

[16] Cordeiro, G.M., Ortega, E.M. and Nadarajah, S., 2010. The Kumaraswamy Weibull distribution with application to failure data. Journal of the Franklin Institute, 347(8), pp.1399-1429.

[17] Cordeiro, G. M., Nadarajah, S. and Ortega, E. M. 2013c. General Results for the Beta Weibull Distribution. Journal of Statistical Computation and Simulation 83 (6): 10821114.

[18] David Osborn and Richard Madey, 1968. The incomplete beta function and its ratio to the complete beta function. Mathematics of Computation Vol. 22, No. 101 pp. 159-162+s16-s32.

[19] Bornemann, Folkmar and Weisstein, Eric W., 2016 Power series From MathWorld-A Wolfram Web Resource. http://mathworld.wolfram.com.

[20] Greenwood, J.A., Landwehr, J.M., Matalas, N.C. and Wallis, J.R., 1979. Probability weighted moments: definition and relation to parameters of several distributions expressable in inverse form. Water Resources Research, 15(5), pp.1049-1054.

[21] Nadarajah, S. and Zografos, K. 2003. Formulas for Renyi Information and Related Measures for Univariate Distributions. Information Sciences 155 (1): 119138 . 
[22] Nadarajah, S., Cordeiro, G.M. and Ortega, E.M., 2012. General results for the Kumaraswamy-G distribution. Journal of Statistical Computation and Simulation, 82(7), pp.951-979.

[23] Dagum C., 1980. The generation and distribution of income, the Lorenz curve and the Gini ratio. Econ Appl XXXIII: 327367.

[24] Song, K.S., 2001. Rnyi information, loglikelihood and an intrinsic distribution measure. Journal of Statistical Planning and Inference, 93(1), pp.51-69.

[25] Kenney J.F., Keeping and E.S., 1962. Mathematics of Statistics, Part 1. Van Nostrand, New Jersey, 3rd edition.

[26] Moors, J.J.A., 1988. A quantile alternative for kurtosis. The statistician, pp.25-32.

[27] Andrews, D.F. and Herzberg, A.M., 1985. Stress-rupture life of kevlar 49/epoxy spherical pressure vessels. In Data (pp. 181-186). Springer New York.

[28] Hosmer, D.W. and Lemeshow, S. and May, S. 2008. Applied Survival Analysis: Regression Modeling of Time to Event Data: Second Edition, John Wiley and Sons Inc., New York, NY.

[29] Shiffman, S., Waters, A.J. and Hickcox, M., 2004. The nicotine dependence syndrome scale: a multidimensional measure of nicotine dependence. Nicotine and Tobacco Research, 6(2), pp.327-348.

[30] Watson, G.S., 1961. Goodness-of-fit tests on a circle. Biometrika, 48(1/2), pp.109-114.

[31] Yusuf, M.U. and Bakar, M.R.B.A., 1739. A Bayesian estimation on right censored survival data with mixture and non-mixture cured fraction model based on Beta-Weibull distribution. In AIP Conference Proceedings (Vol. 20079, No. 2016).

[32] Pescim, R. R., Cordeiro, G. M., Demetrio, C. G., Ortega, E. M. and Nadarajah, S. 2012. The New Class of Kummer Beta Generalized Distributions. Statistics and Operations Research Transactions (SORT) 36 (2): 153(180)

[33] Nadarajah, S. and Zografos, K. 2003. Formulas for Renyi Information and Related Measures for Univariate Distributions. Information Sciences 155 (1): 119(138)

[34] Zografos, K. and Nadarajah, S. 2005. Expressions for Renyi and Shannon Entropies for Multivariate Distributions. Statistics and Probability Letters $71(1)$ : 